\title{
ON STOCHASTIC EVOLUTION EQUATIONS WITH NON-LIPSCHITZ COEFFICIENTS
}

\author{
XICHENG ZHANG \\ Fakultät für Mathematik, Universität Bielefeld \\ Postfach 100131, D-33501 Bielefeld, Germany \\ Department of Mathematics, Huazhong University of Science and Technology, \\ Wuhan, Hubei 4300\%4, P.R.China \\ Email: hiverzxc@hotmail.com
}

\begin{abstract}
In this paper, we study the existence and uniqueness of solutions for several classes of stochastic evolution equations with non-Lipschitz coefficients, that is, backward stochastic evolution equations, stochastic Volterra type evolution equations and stochastic functional evolution equations. In particular, the results can be used to treat a large class of quasi-linear stochastic equations, which includes the reaction diffusion and porous medium equations.
\end{abstract}

\section{Contents}

1. Introduction

2. Framework and Preliminaries

3. Stochastic Evolution Equations in Banach Spaces

4. Backward Stochastic Evolution Equations

5. Stochastic Functional Integral Evolution Equations

References

\section{INTRODUCTION}

Let $\mathcal{O}$ be a bounded open subset of $\mathbb{R}^{d}$. Consider the following stochastic porous medium equation with Dirichlet boundary condition:

$$
\left\{\begin{array}{l}
\mathrm{d} u_{t}=\left|w_{t}\right| \cdot \Delta\left(\left|u_{t}\right|^{p-2} u_{t}\right) \mathrm{d} t+\mathrm{d} w_{t} \\
u_{t}(x)=0, \quad x \in \partial \mathcal{O}, t>0 \\
u_{0}=\phi \in L^{p}(\mathcal{O})
\end{array}\right.
$$

where $p \geqslant 2, \Delta$ is the usual Laplace operator and $\left\{w_{t}, t \geqslant 0\right\}$ is a one dimensional standard Brownian motion. This is a degenerate non-linear stochastic partial differential equation. Notice that the degeneracy may be caused by $w_{t}=0$ and $u_{t}=0$. In the deterministic case, it is well known that porous medium equations can be written as abstract monotone operator equations(cf. [33] [28]). Thus, in the stochastic case, it can fall into a class of stochastic evolution equations studied by Krylov-Rozovskii [16. More discussions about the stochastic porous medium equation are referred to [8] [24] [26].

Key words and phrases. Stochastic Reaction Diffusion Equation, Stochastic Porous Medium Equation, Stochastic Evolution Equation, Backward Stochastic Evolution Equation, Stochastic Functional Integral Evolution Equation. 
On the other hand, let us consider the following stochastic reaction diffusion equation:

$$
\left\{\begin{array}{l}
\mathrm{d} u_{t}=\left|w_{t}\right| \cdot\left(\Delta u_{t}-\left|u_{t}\right|^{p-2} \cdot u_{t}\right) \mathrm{d} t+\left(w_{t} \cdot u_{t}\right) \mathrm{d} w_{t} \\
u_{t}(x)=0, \quad x \in \partial \mathcal{O}, t>0 \\
u_{0}=\phi \in L^{2}(\mathcal{O})
\end{array}\right.
$$

where $p \geqslant 2$. Usually, one wants to find an adapted process $u$ such that for almost all $w$

$$
u .(w) \in L^{2}\left([0, T], H_{0}^{1}(\mathcal{O})\right) \cap L^{p}([0, T] \times \mathcal{O}) \cap C\left([0, T], L^{2}(\mathcal{O})\right),
$$

and (2) holds in the generalized sense, where $H_{0}^{1}(\mathcal{O})$ is the usual Sobolev space.

However, from the well known results, it seems that one cannot solve Eq.(11) and Eq.(2) because of the existence of $\left|w_{t}\right|$ in front of the Laplace operator. One of the main purposes in this paper is to extend the well known results in [16] [11] so that we can solve Eq.(1) and Eq.(2) in the generalized sense for almost all path $w_{t}$.

In the present paper, we shall work on the framework of evolution triple. This is crucial for treating a wide class of quasi-linear stochastic partial differential equations(including reaction diffusion equations and porous medium equations). We now recall some wellknown results in this direction. In [19] [20], Pardoux considered linear stochastic partial differential equations(SPDEs) using the monotonicity method. In [16], basing on their established Itô's formula, Krylov and Rozovskii proved a more general result under some monotonicity or dissipative conditions. This classic work was later extended in several aspects: to stochastic evolution equations(SEEs) driven by general (discontinuous) martingales in [10], to SEEs with coercivity constants depending on $t$ in [11], to SEEs related to some Orlicz spaces in [24]. All these works are based on Galerkin's approximation. It should be remarked that the semigroup method is another main tool in the theory of semi-linear SPDEs (cf. [9] [6] [7] [15] [34] etc.). In order to solve Eq.(11), we need to deal with SEEs with random coercivity coefficients. This is our first goal, and will be done in Section 3 after some preliminaries of Section 2. Here, some stopping times techniques will be used. As said above, since porous medium equations can be written as monotone operator equations, we remark that solving Eq.(1) will follow from our Theorem 3.6 below by a standard argument (cf. [28] [26]). As for Eq.(2), one only needs to choose the corresponding spaces in Theorem 3.6 as follows:

$$
\mathbb{X}=H_{0}^{1}(\mathcal{O}) \cap L^{p}(\mathcal{O}), \quad \mathbb{H}=L^{2}(\mathcal{O}), \quad \mathbb{X}^{*}=H^{-1}(\mathcal{O})+L^{\frac{p}{p-1}}(\mathcal{O}),
$$

where $H^{-1}(\mathcal{O})$ is the dual space of $H_{0}^{1}(\mathcal{O})$.

The second aim is to prove the existence and uniqueness of solutions to backward stochastic evolution equations. Since Pardoux and Peng in [21] proved the existence and uniqueness of solutions to nonlinear backward stochastic differential equations(BSDEs), the theory of BSDEs has already been developed extensively. It is well known that BSDEs can be applied to the studies of stochastic controls, mathematics finances, deterministic PDEs, etc.. Meanwhile, backward SPDEs have also been studied in [13] [23] etc.. In these works, the authors mainly concentrated on the semilinear BSPDEs. The second aim in this paper is to prove the existence and uniqueness of solutions to BSEEs with non-Lipschitz coefficients in the framework of evolution triple. Thus, it can be used to deal with a large class of quasi linear BSPDE. We remark that Mao in [17] has already studied BSDEs with non-Lipschitz coefficients. This is the content of Section 4.

The third aim is to study the stochastic functional integral evolution equations with non-Lipschitz coefficients, which in particular includes a class of stochastic Volterra type evolution equations. Stochastic Volterra equations driven by Brownian motion were first studied by Berger-Mizel [3]. Later, Protter [22] proved the existence and uniqueness of 
stochastic Volterra equations driven by general semimartingales. Recently, Wang and the present author in [31] studied the the existence and uniqueness of stochastic Volterra equations with singular kernels and non-Lipschitz coefficients. About the stochastic functional differential equations, Mohammend's book [18] is one of the main references. In [29], using the evolution semigroup approach, the authors studied the existence, uniqueness and asymptotic behavior of mild solutions to stochastic semilinear functional differential equations in Hilbert spaces. In our proof of Section 5, the main tool is the usual Picard iteration. As above, the results in Section 5 can be also used to deal with a class of quasi linear stochastic functional partial differential equations.

\section{Framework and Preliminaries}

In this section we present a general setting in which we can deal with a large class of non-linear stochastic partial differential equations, and also recall the powerful Itô formula and a nonlinear Gronwall type inequality (Bihari's inequality) for treating non-Lipschitz equations.

Let $\mathbb{X}$ be a reflexive and separable Banach space, which is densely injected in a separable Hilbert space $\mathbb{H}$. Identifying $\mathbb{H}$ with its dual we get

$$
\mathbb{X} \subset \mathbb{H} \simeq \mathbb{H}^{*} \subset \mathbb{X}^{*}
$$

where the star ${ }^{*}$ ' denotes the dual spaces.

Assume that the norm in $\mathbb{X}$ is given by

$$
\|x\|_{\mathbb{X}}:=\|x\|_{1, \mathbb{X}}+\|x\|_{2, \mathbb{X}}, \quad x \in \mathbb{X} .
$$

Denote by $\mathbb{X}_{i}, i=1,2$ the completions of $\mathbb{X}$ with respect to the norms $\|\cdot\|_{i, \mathbb{X}}=:\|\cdot\|_{\mathbb{X}_{i}}$. Let us also assume that both spaces are reflexive and embedded in $\mathbb{H}$. Notice that $\mathbb{X}^{*}$ is the set of all $f=f_{1}+f_{2}, f_{i} \in \mathbb{X}_{i}^{*}, i=1,2$ such that $\left\|f_{i}\right\|_{\mathbb{X}_{i}^{*}}<+\infty$ and the norm of $f$ is also given by

$$
\|f\|_{\mathbb{X}^{*}}=\inf _{f=f_{1}+f_{2}}\left(\left\|f_{1}\right\|_{\mathbb{X}_{1}^{*}}+\left\|f_{2}\right\|_{\mathbb{X}_{2}^{*}}\right) .
$$

In the following, the dual pairs of $\left(\mathbb{X}, \mathbb{X}^{*}\right)$ and $\left(\mathbb{X}_{i}, \mathbb{X}_{i}^{*}\right), i=1,2$ are denoted respectively by

$$
[\cdot, \cdot]_{\mathbb{X}}, \quad[\cdot, \cdot]_{\mathbb{X}_{i}}, \quad i=1,2 .
$$

Then, for any $x \in \mathbb{X}$ and $f=f_{1}+f_{2} \in \mathbb{X}^{*}$,

$$
[x, f]_{\mathbb{X}}=\left[x, f_{1}\right]_{\mathbb{X}_{1}}+\left[x, f_{2}\right]_{\mathbb{X}_{2}} .
$$

We remark that if $f \in \mathbb{H}$ and $x \in \mathbb{X}$, then

$$
[x, f]_{\mathbb{X}}=[x, f]_{\mathbb{X}_{1}}=[x, f]_{\mathbb{X}_{2}}=\langle x, f\rangle_{\mathbb{H}},
$$

where $\langle\cdot, \cdot\rangle_{\mathbb{H}}$ stands for the inner product in $\mathbb{H}$.

Let $\left(\Omega, \mathcal{F},\left(\mathcal{F}_{t}\right)_{t \geqslant 0}, P\right)$ be a complete separable filtration probability space, and $Q$ a nonnegative definite and symmetric bounded linear operator on another Hilbert space $\mathbb{U}$. A cylindrical $Q$-Wiener process $\{W(t), t \geqslant 0\}$ defined on $(\Omega, \mathcal{F}, P)$ is given and assumed to be adapted to $\left(\mathcal{F}_{t}\right)_{t \geqslant 0}(\mathrm{cf}$. [9] $)$. In the following we shall only consider the case of $Q \equiv I$ for simplicity. Let $L_{2}(\mathbb{U}, \mathbb{H})$ denote the Hilbert space consisting of all the Hilbert-Schmidt operators from $\mathbb{U}$ to $\mathbb{H}$, where the norm is denoted by $\|\cdot\|_{L_{2}(\mathbb{U}, \mathbb{H})}$, and the inner product by $\langle\cdot, \cdot,\rangle_{L_{2}(\mathbb{U}, \mathbb{H})}$.

Fix $T>0$. Let $\mathcal{M}$ be the total of progressively measurable subsets of $[0, T] \times \Omega$. The following Itô formula is taken from Gyöngy-Krylov [12]. 
Theorem 2.1. Let $X_{0}$ be an $\mathcal{F}_{0}$-measurable $\mathbb{H}$-valued random variable. Let

$$
Y_{i}:[0, T] \times \Omega \mapsto \mathbb{X}_{i}^{*} \in \mathcal{M} / \mathcal{B}\left(\mathbb{X}_{i}^{*}\right), \quad i=1,2,
$$

and $M$ an $\mathbb{H}$-valued continuous locally square integrable martingale starting form zero. Let $\lambda_{1}, \lambda_{2}$ be two $\mathcal{M} / \mathcal{B}(\mathbb{R})$-measurable real valued processes such that for $(\mathrm{d} t \times \mathrm{d} P)$-almost all $(t, \omega), \lambda_{1}(t, \omega), \lambda_{2}(t, \omega)>0$. Assume that for some $q_{1}, q_{2}>1$ and for almost all $\omega$,

$$
\lambda_{i}(\cdot, \omega) \in L^{1}([0, T], \mathrm{d} t), \quad Y_{i}(\cdot, \omega) \cdot \lambda_{i}^{-\frac{1}{q_{i}}}(\cdot, \omega) \in L^{\frac{q_{i}}{q_{i}-1}}\left([0, T], \mathrm{d} t ; \mathbb{X}^{*}\right), \quad i=1,2 .
$$

Define an $\mathbb{X}^{*}$-valued process by

$$
X(t):=X_{0}+\int_{0}^{t} Y_{1}(s) \mathrm{d} s+\int_{0}^{t} Y_{2}(s) \mathrm{d} s+M(t) .
$$

If there exists a $(\mathrm{d} t \times \mathrm{d} P)$-version $\tilde{X}$ of $X$ such that for almost all $\omega$,

$$
\tilde{X}(\cdot, \omega) \cdot \lambda_{i}^{\frac{1}{q_{i}}}(\cdot, \omega) \in L^{q_{i}}\left([0, T], \mathrm{d} t ; \mathbb{X}_{i}\right), \quad i=1,2,
$$

then for almost all $\omega$,

(i) $[0, T] \ni t \mapsto X(t, \omega) \in \mathbb{H}$ is continuous;

(ii) for all $t \in[0, T]$

$$
\begin{aligned}
\|X(t, \omega)\|_{\mathbb{H}}^{2}= & \left\|X_{0}(\omega)\right\|_{\mathbb{H}}^{2}+2 \int_{0}^{t}\left[\tilde{X}(s, \omega),\left(Y_{1}+Y_{2}\right)(s, \omega)\right]_{\mathbb{X}} \mathrm{d} s \\
& +2 \int_{0}^{t}\langle X(s), \mathrm{d} M(s)\rangle_{\mathbb{H}}(\omega)+\langle M\rangle(t, \omega),
\end{aligned}
$$

where $\langle\cdot\rangle$ denotes the quadratic variation of $\mathbb{H}$-valued martingale.

Proof. Set $N_{i}(t):=\int_{0}^{t} \lambda_{i}^{\frac{1}{q_{i}}}(s) \mathrm{d} s$ and $\tilde{Y}_{i}(t):=Y_{i}(t) \cdot \lambda_{i}^{-\frac{1}{q_{i}}}(s), i=1,2$. Then

$$
X(t)=X_{0}+\int_{0}^{t} \tilde{Y}_{1}(s) \mathrm{d} N_{1}(s)+\int_{0}^{t} \tilde{Y}_{2}(s) \mathrm{d} N_{2}(s)+M(t) .
$$

By the assumptions and Hölder's inequality, we have for almost all $\omega$,

$$
\begin{aligned}
& \tilde{Y}_{i}(\cdot, \omega) \in L^{1}\left([0, T], \mathrm{d} N_{i} ; \mathbb{X}_{i}^{*}\right), \quad i=1,2, \\
& \tilde{X}(\cdot, \omega) \in \cap_{i=1,2} L^{1}\left([0, T], \mathrm{d} N_{i} ; \mathbb{X}_{i}\right) .
\end{aligned}
$$

Moreover, by Hölder's inequality we have for $i=1,2$

$$
\begin{aligned}
& \int_{0}^{T}\left\|\tilde{Y}_{i}(t, \omega)\right\|_{\mathbb{X}_{i}^{*}} \cdot\|\tilde{X}(t, \omega)\|_{\mathbb{X}_{i}} \mathrm{~d} N_{i}(t) \\
= & \int_{0}^{T}\left\|Y_{i}(t, \omega)\right\|_{\mathbb{X}_{i}^{*}} \lambda_{i}^{-\frac{1}{q_{i}}}(t) \cdot\|\tilde{X}(t, \omega)\|_{\mathbb{X}_{i}} \lambda_{i}^{\frac{1}{q_{i}}}(t) \mathrm{d} t \\
\leqslant & \left(\int_{0}^{T}\left\|Y_{i}(t, \omega)\right\|_{\mathbb{X}_{i}^{*}}^{\frac{q_{i}}{q_{i}-1}} \lambda_{i}^{-\frac{1}{q_{i}-1}}(t) \mathrm{d} t\right)^{\frac{q_{i}-1}{q_{i}}} \\
& \times\left(\int_{0}^{T}\|\tilde{X}(t, \omega)\|_{\mathbb{X}_{i}^{*}}^{q_{i}^{*}} \lambda_{i}(t) \mathrm{d} t\right)^{\frac{1}{q_{i}}}<+\infty .
\end{aligned}
$$

Thus, we can prove this Theorem along the same lines as in the proof of [12, Theorem 2] (see also [16] [27] [26]). We omit the details. 
We now recall the following Bihari inequality(cf. [4]). A multi-parameter version with jump was proved in [36].

Lemma 2.2. Let $\rho: \mathbb{R}^{+} \mapsto \mathbb{R}^{+}$be a continuous and non-decreasing function. If $g(s)$ and $\lambda(s)$ are two strictly positive functions on $\mathbb{R}^{+}$such that for some $g_{0}>0$

$$
g(t) \leqslant g_{0}+\int_{0}^{t} \lambda(s) \cdot \rho(g(s)) \mathrm{d} s, \quad t \geqslant 0,
$$

then

$$
g(t) \leqslant G^{-1}\left(G\left(g_{0}\right)+\int_{0}^{t} \lambda(s) \mathrm{d} s\right)
$$

where $G(x):=\int_{x_{0}}^{x} \frac{1}{\rho(y)} \mathrm{d} y$ is well defined for some $x_{0}>0$, and $G^{-1}$ is the inverse function of $G$.

In particular, if $g_{0}=0$ and for some $\varepsilon>0$

$$
\int_{0}^{\varepsilon} \frac{1}{\rho(x)} \mathrm{d} x=+\infty
$$

then $g(t) \equiv 0$.

Remark 2.3. The typical concave functions satisfying (3) are given by $\rho_{k}(x), k=$ $1,2, \cdots$,

$$
\rho_{k}(x):= \begin{cases}c_{0} \cdot x \cdot \Pi_{j=1}^{k} \log ^{j} x^{-1}, & x \leqslant \eta \\ c_{0} \cdot \eta \cdot \Pi_{j=1}^{k} \log ^{j} \eta^{-1}+c_{0} \cdot \rho_{k}^{\prime}(\eta-) \cdot(x-\eta), & x>\eta,\end{cases}
$$

where $\log ^{j} x^{-1}:=\log \log \cdots \log x^{-1}$ and $c_{0}>0,0<\eta<1 / e^{k}$.

In the sequel, we use the following convention: $c_{0}, c_{1}, \cdots$ will denote positive constants whose values may change in different occasions. Moreover, the following Young inequality will be used frequently: Let $a, b>0$ and $\alpha, \beta>1$ satisfying $\frac{1}{\alpha}+\frac{1}{\beta}=1$, then for any $\varepsilon>0$

$$
a b \leqslant \varepsilon a^{\alpha}+\frac{b^{\beta}}{(\alpha \varepsilon)^{\beta / \alpha} \beta} .
$$

For the simplicity of notations, we also write

$$
\mathfrak{A}:=([0, T] \times \Omega, \mathcal{B}([0, T]) \times \mathcal{F}, \mathrm{d} t \times \mathrm{d} P)
$$

and

$$
\mathfrak{A}_{a}:=([0, T] \times \Omega, \mathcal{M}, \mathrm{d} t \times \mathrm{d} P) .
$$

We now introduce three evolution operators used in the present paper:

$$
\begin{aligned}
A_{i}:[0, T] \times \Omega \times \mathbb{X}_{i} \mapsto \mathbb{X}_{i}^{*} & \in \mathcal{M} \times \mathcal{B}\left(\mathbb{X}_{i}\right) / \mathcal{B}\left(\mathbb{X}_{i}^{*}\right), \quad i=1,2, \\
B:[0, T] \times \Omega \times \mathbb{X} \mapsto L_{2}(\mathbb{U}, \mathbb{H}) & \in \mathcal{M} \times \mathcal{B}(\mathbb{X}) / \mathcal{B}\left(L_{2}(\mathbb{U}, \mathbb{H})\right) .
\end{aligned}
$$

In the following, for the sake of simplicity, we write

$$
A=A_{1}+A_{2} \in \mathbb{X}^{*}
$$

Assume that

(H1) (Hemicontinuity) For any $(t, \omega) \in[0, T] \times \Omega$ and $x, y, z \in \mathbb{X}$, the mapping

$$
[0,1] \ni \varepsilon \mapsto[x, A(t, \omega, y+\varepsilon z)]_{\mathbb{X}}
$$

is continuous. 
(H2) (Weak monotonicity) There exists $0 \leqslant \lambda_{0} \in L^{1}(\mathfrak{A})$ such that for all $x, y \in \mathbb{X}$ and $(t, \omega) \in[0, T] \times \Omega$

$$
\begin{gathered}
2[x-y, A(t, \omega, x)-A(t, \omega, y)]_{\mathbb{X}}+\|B(t, \omega, x)-B(t, \omega, y)\|_{L_{2}(\mathbb{U}, \mathbb{H})}^{2} \\
\leqslant \lambda_{0}(t, \omega) \cdot\|x-y\|_{\mathbb{H}}^{2} .
\end{gathered}
$$

(H3) (Weak coercivity) There exist $q_{1}, q_{2} \geqslant 2, c_{1}>0$ and positive functions $\lambda_{1}, \lambda_{3}, \lambda_{3}, \xi \in$ $L^{1}(\mathfrak{A})$ satisfying that for almost all $(t, \omega)$

$$
0 \leqslant \lambda_{0}(t, \omega)<c_{1} \cdot\left(\lambda_{1}(t, \omega) \wedge \lambda_{2}(t, \omega)\right)
$$

and

$$
(t, \omega) \mapsto \lambda_{i}(t, \omega) \cdot e^{\frac{q_{i}-2}{2} \int_{0}^{t} \lambda_{0}(s, \omega) \mathrm{d} s} \in L^{1}(\mathfrak{A}), \quad i=1,2,
$$

where $\lambda_{0}$ is same as in (H2), and such that for all $x \in \mathbb{X}$ and $(t, \omega) \in[0, T] \times \Omega$

$$
\begin{aligned}
& 2[x, A(t, \omega, x)]_{\mathbb{X}}+\|B(t, \omega, x)\|_{L_{2}(\mathbb{U}, \mathbb{H})}^{2} \\
\leqslant & -\sum_{i=1,2}\left(\lambda_{i}(t, \omega) \cdot\|x\|_{\mathbb{X}_{i}}^{q_{i}}\right)+\lambda_{3}(t, \omega) \cdot\|x\|_{\mathbb{H}}^{2}+\xi(t, \omega) .
\end{aligned}
$$

(H4) (Boundedness) There exist $c_{A_{i}}>0$ and $0 \leqslant \eta_{i} \in L^{\frac{q_{i}}{q_{i}-1}}(\mathfrak{A}), i=1,2$ such that for all $x \in \mathbb{X}$ and $(t, \omega) \in[0, T] \times \Omega$

$$
\left\|A_{i}(t, \omega, x)\right\|_{\mathbb{X}_{i}^{*}} \leqslant \eta_{i}(t, \omega) \cdot \lambda_{i}^{\frac{1}{q_{i}}}(t, \omega)+c_{A_{i}} \cdot \lambda_{i}(t, \omega) \cdot\|x\|_{\mathbb{X}_{i}}^{q_{i}-1}, \quad i=1,2,
$$

where $q_{1}$ and $q_{2}$ are same as in (H3).

In order to emphasize $\lambda_{i}, \xi$ and $q_{i}, \eta_{i}$ below, we shall say that

$$
(A, B) \text { satisfies } \mathscr{H}\left(\lambda_{0}, \lambda_{1}, \lambda_{2}, \lambda_{3}, \xi, \eta_{1}, \eta_{2}, q_{1}, q_{2}\right) \text {. }
$$

If there are no special declarations, we always suppose that $q_{i} \geqslant 2, c_{1}, c_{A_{i}}>0, \eta_{i} \in$ $L^{\frac{q_{i}}{q_{i}-1}}(\mathfrak{A}), i=1,2$ and $\lambda_{i}, \xi \in L^{1}(\mathfrak{A}), i=0,1,2,3$ are strictly positive functions, and (6) - (17) hold.

Remark 2.4. By (H3), (H4) and Young's inequality (5), it follows that for any $x \in \mathbb{X}$ and $(t, \omega) \in[0, T] \times \Omega$

$$
\begin{aligned}
\|B(t, \omega, x)\|_{L_{2}(\mathbb{U}, \mathbb{H})}^{2} \leqslant & 2 \sum_{i=1,2}\left(c_{A_{i}} \cdot \lambda_{i}(t, \omega) \cdot\|x\|_{\mathbb{X}_{i}}^{q_{i}}+\eta_{i}(t, \omega) \cdot \lambda_{i}^{\frac{1}{q_{i}}}(t, \omega) \cdot\|x\|_{\mathbb{X}_{i}}\right) \\
& +\lambda_{3}(t, \omega) \cdot\|x\|_{\mathbb{H}}^{2}+\xi(t, \omega) \\
\leqslant & \sum_{i=1,2}\left(c_{B} \cdot \lambda_{i}(t, \omega) \cdot\|x\|_{\mathbb{X}_{i}}^{q_{i}}+\eta_{i}^{\frac{q_{i}}{q_{i}-1}}(t, \omega)\right) \\
& +\lambda_{3}(t, \omega) \cdot\|x\|_{\mathbb{H}}^{2}+\xi(t, \omega),
\end{aligned}
$$

where $c_{B}>1$ only depends on $c_{A_{i}}$ and $q_{i}, i=1,2$.

The following lemma is well known(cf. [16]).

Lemma 2.5. Let $(A, 0)$ satisfy $\mathscr{H}\left(0, \lambda_{1}, \lambda_{2}, \lambda_{3}, \xi, \eta_{1}, \eta_{2}, q_{1}, q_{2}\right)$, and $0 \leqslant \tau \leqslant T$ a bounded random variable. Let $X$ and $Y_{i}(i=1,2)$ be respectively $\mathbb{X}$ and $\mathbb{X}_{i}^{*}$-valued measurable processes with

$$
1_{[0, \tau]}(\cdot) \cdot \lambda_{i}^{\frac{1}{q_{i}-1}} \cdot X \in L^{q_{i}-1}\left(\mathfrak{A} ; \mathbb{X}_{i}\right), \quad{ }_{6} 1_{[0, \tau]}(\cdot) \cdot Y_{i} \in L^{1}\left(\mathfrak{A} ; \mathbb{X}_{i}^{*}\right), \quad i=1,2 .
$$


Assume that for any $\mathbb{X}$-valued measurable process $\Phi$ satisfying

$$
1_{[0, \tau]}(\cdot) \cdot \lambda_{i}^{\frac{1}{q_{i}-1}} \cdot \Phi \in L^{q_{i}-1}\left(\mathfrak{A} ; \mathbb{X}_{i}\right), \quad i=1,2,
$$

it holds

$$
\mathbb{E}\left(\int_{0}^{\tau}[X(s)-\Phi(s), Y(s)-A(s, \Phi(s))]_{\mathbb{X}} \mathrm{d} s\right) \leqslant 0
$$

where $Y=Y_{1}+Y_{2} \in \mathbb{X}^{*}$.

Then $Y(t, \omega)=A(t, \omega, X(t, \omega))$ for almost all $(t, \omega)$.

Proof. For any $\varepsilon \in(0,1)$ and $\mathbb{X}$-valued bounded measurable process $\phi$, letting $\Phi=X-\varepsilon \phi$ in (8) and dividing both sides by $\varepsilon$, we get

$$
\mathbb{E}\left(\int_{0}^{\tau}[\phi(s), Y(s)-A(s, X(s)-\varepsilon \phi(s))]_{\mathbb{X}} \mathrm{d} s\right) \leqslant 0 .
$$

By $(\mathbf{H 4})$ and the assumptions, we have

$$
1_{[0, \tau]}(\cdot) \cdot\left(\|Y(\cdot)\|_{\mathbb{X}^{*}}+\sup _{\varepsilon \in(0,1)}\|A(\cdot, X(\cdot)-\varepsilon \phi(\cdot))\|_{\mathbb{X}^{*}}\right) \in L^{1}(\mathfrak{A}) .
$$

Hence, by $(\mathbf{H 1})$ and the dominated convergence theorem

$$
\mathbb{E}\left(\int_{0}^{\tau}[\phi(s), Y(s)-A(s, X(s))]_{\mathbb{X}} \mathrm{d} s\right) \leqslant 0 .
$$

By changing $\phi$ to $-\phi$ and the arbitrariness of $\phi$, we conclude that $Y=A(\cdot, X)$.

The following lemma is simple and will be used in Section 4. A short proof is provided here for the reader's convenience.

Lemma 2.6. Let $(S, \mathcal{S})$ be a measurable space. Let $X: \mathbb{R}^{d} \times S \mapsto \mathbb{R}^{d}$ be a measurable field. Assume that for every $s \in S, \mathbb{R}^{d} \ni x \mapsto X(x, s) \in \mathbb{R}^{d}$ is a homeomorphism. Then, the inverse $(x, s) \mapsto X^{-1}(x, s)$ is also a measurable field, i.e.: for each $x \in \mathbb{R}^{d}, X^{-1}(x, \cdot)$ is $\mathcal{S} / \mathcal{B}\left(\mathbb{R}^{d}\right)$-measurable.

Proof. Fix $x \in \mathbb{R}^{d}$. It suffices to prove that for any bounded open set $U \subset \mathbb{R}^{d}$

$$
S_{1}:=\left\{s: X^{-1}(x, s) \in \bar{U}\right\} \in \mathcal{S}
$$

where $\bar{U}$ denotes the closure of $U$ in $\mathbb{R}^{d}$.

Let $Q$ be the set of rational points in $\mathbb{R}^{d}$. Then

$$
S_{1}=\cap_{k=1}^{\infty} \cup_{y \in Q \cap U}\left\{s:\|X(y, s)-x\|_{\mathbb{R}^{d}}<1 / k\right\}=: S_{2} .
$$

In fact, if $s \in S_{1}$, then there is a $y \in \bar{U}$ such that $x=X(y, s)$. Since $U$ is open and $X(\cdot, s)$ is continuous, there exists a sequence $y_{n} \in U \cap Q$ such that $y_{n} \rightarrow y$ and $X\left(y_{n}, s\right) \rightarrow X(y, s)=x$. So, $s \in S_{2}$. On the other hand, if $s \in S_{2}$, then there is a sequence $y_{n} \in U \cap Q$ such that $\lim _{n \rightarrow \infty}\left\|X\left(y_{n}, s\right)-x\right\|_{\mathbb{R}^{d}}=0$, and so $y_{n} \rightarrow X^{-1}(x, s) \in \bar{U}$. (9) now follows from (10). 


\section{Stochastic Evolution Equations in Banach Spaces}

In this section, we consider the following stochastic evolution equation:

$$
\left\{\begin{array}{l}
\mathrm{d} X(t)=A(t, X(t)) \mathrm{d} t+B(t, X(t)) \mathrm{d} W(t), \\
X(0)=X_{0} \in \mathbb{H},
\end{array}\right.
$$

where $(A, B)$ satisfies $\mathscr{H}\left(\lambda_{0}, \lambda_{1}, \lambda_{2}, \lambda_{3}, \xi, \eta_{1}, \eta_{2}, q_{1}, q_{2}\right)$. Here and after, one should keep in mind that $A=A_{1}+A_{2} \in \mathbb{X}^{*}$, where $A_{1} \in \mathbb{X}_{1}^{*}, A_{2} \in \mathbb{X}_{2}^{*}$.

Set

$$
H(t, \omega):=\int_{0}^{t} \lambda_{3}(s, \omega) \mathrm{d} s
$$

and define

$$
\theta_{t}(\omega):=\inf \{s \in[0, T]: H(s, \omega) \geqslant t\} .
$$

Then $t \mapsto \theta_{t}$ are continuous stopping times, and $\theta_{t} \uparrow T$ as $t \uparrow \infty$. Here, $\inf \{\emptyset\}=T$ by convention.

Set for each $m \in \mathbb{N}$

$$
\mu^{m}(\mathrm{~d} t \times \mathrm{d} \omega):=1_{\left\{t \leqslant \theta_{m}(\omega)\right\}}(\mathrm{d} t \times \mathrm{d} P),
$$

and define the completed measurable spaces

$$
\mathfrak{M}^{m}:=\overline{([0, T] \times \Omega, \mathcal{B}([0, T]) \times \mathcal{F}}^{\mu^{m}}
$$

and

$$
\mathfrak{M}_{a}^{m}:=\overline{([0, T] \times \Omega, \mathcal{M})} \mu^{m} .
$$

We introduce the following stochastic Banach spaces for later use: for each $m \in \mathbb{N}$

$$
\begin{aligned}
\mathbb{K}_{1, i}^{m} & :=L^{\frac{q_{i}}{q_{i}-1}}\left(\mathfrak{M}^{m}, \lambda_{i}^{-\frac{1}{q_{i}-1}}(t, \omega) \cdot \mu^{m}(\mathrm{~d} t \times \mathrm{d} \omega) ; \mathbb{X}_{i}^{*}\right), \quad i=1,2, \\
\mathbb{K}_{2, i}^{m} & :=L^{q_{i}}\left(\mathfrak{M}^{m}, \lambda_{i}(t, \omega) \cdot \mu^{m}(\mathrm{~d} t \times \mathrm{d} \omega) ; \mathbb{X}_{i}\right), \quad i=1,2, \\
\mathbb{K}_{3}^{m} & :=L^{2}\left(\mathfrak{M}_{a}^{m}, \mu^{m}(\mathrm{~d} t \times \mathrm{d} \omega) ; L_{2}(\mathbb{U}, \mathbb{H})\right), \\
\mathbb{K}_{4}^{m} & :=L^{2}\left(\mathfrak{M}^{m}, \mu^{m}(\mathrm{~d} t \times \mathrm{d} \omega) ; \mathbb{H}\right), \\
\mathbb{K}_{5}^{m} & :=L^{2}\left(\mathfrak{M}^{m}, \lambda_{3}(t, \omega) \cdot \mu^{m}(\mathrm{~d} t \times \mathrm{d} \omega) ; \mathbb{H}\right),
\end{aligned}
$$

where the norms are defined in a natural manner, and denoted by $\|\cdot\|_{\mathbb{K}}$, where $\mathbb{K}$ stands for the above spaces. For instance,

$$
\|X\|_{\mathbb{K}_{2, i}^{m}}:=\left[\mathbb{E}\left(\int_{0}^{\theta_{m}}\|X(t)\|_{\mathbb{X}_{i}}^{q_{i}} \cdot \lambda_{i}(t) \mathrm{d} t\right)\right]^{1 / q_{i}}, \quad i=1,2 .
$$

Remark 3.1. If $\lambda_{3}$ is non-random, then for some $m$ sufficiently large, $\theta_{m} \equiv T$. In this case, we shall omit the superscript ' $m$ ' of $\mathbb{K}^{m}$.

We need the following lemma.

Lemma 3.2. (i) $\mathbb{K}_{i, j}^{m}, i, j=1,2$ and $\mathbb{K}_{3}^{m}, \mathbb{K}_{4}^{m}, \mathbb{K}_{5}^{m}$ are separable and reflexive Banach spaces.

(ii) For any $Y \in \mathbb{K}_{1, i}^{m}$, we have $\mathbb{E}\left(\int_{0}^{\theta_{m}}\|Y(t)\|_{\mathbb{X}_{i}^{*}} \mathrm{~d} t\right) \leqslant c_{0} \cdot\|Y\|_{\mathbb{K}_{1, i}^{m}}$, where $i=1$ or 2 .

(iii) Let $\left\{Y_{n}, n \in \mathbb{N}\right\}$ weakly converge to $Y$ in $\mathbb{K}_{1, i}^{m}$, then for any $X \in \mathbb{K}_{2, i}^{m}$

$$
\lim _{n \rightarrow \infty} \mathbb{E}\left(\int_{0}^{\theta_{m}}\left[X(t), Y_{n}(t)\right]_{\mathbb{X}_{i}} \mathrm{~d} t\right)=\mathbb{E}\left(\int_{0}^{\theta_{m}}[X(t), Y(t)]_{\mathbb{X}_{i}} \mathrm{~d} t\right),
$$

where $i=1$ or 2 . 
(iv) Let $\left\{X_{n}, n \in \mathbb{N}\right\}$ weakly converge to $X$ in $\mathbb{K}_{2, i}^{m}$, then for any $Y \in \mathbb{K}_{1, i}^{m}$

$$
\lim _{n \rightarrow \infty} \mathbb{E}\left(\int_{0}^{\theta_{m}}\left[X_{n}(t), Y(t)\right]_{\mathbb{X}_{i}} \mathrm{~d} t\right)=\mathbb{E}\left(\int_{0}^{\theta_{m}}[X(t), Y(t)]_{\mathbb{X}_{i}} \mathrm{~d} t\right),
$$

where $i=1$ or 2 . Moreover, if $\left\{X_{n}, n \in \mathbb{N}\right\}$ also weakly converges to $\bar{X}$ in $\mathbb{K}_{5}^{m}$, then $\bar{X}(t, \omega)=X(t, \omega)$ for $\mu^{m}$-almost all $(t, \omega)$.

(v) Define a linear operator from $\mathbb{K}_{3}^{m}$ to $\mathbb{K}_{4}^{m}$ as

$$
J(G):=\int_{0}^{\cdot \wedge \theta_{m}} G(s) \mathrm{d} W(s),
$$

then $J$ is a continuous linear operator. In particular, $J$ is continuous with respect to the weak topologies.

Proof. (i). It follows from the separabilities and reflexivities of $\mathbb{X}_{i}, \mathbb{X}_{i}^{*}, i=1,2$, and $\mathbb{H}, L_{2}(\mathbb{U}, \mathbb{H})$.

(ii). By Hölder's inequality we have

$$
\begin{aligned}
\mathbb{E}\left(\int_{0}^{\theta_{m}}\|Y(t)\|_{\mathbb{X}_{i}^{*}} \mathrm{~d} t\right) & =\mathbb{E}\left(\int_{0}^{\theta_{m}}\|Y(t)\|_{\mathbb{X}_{i}^{*}} \lambda_{2}^{-1 / q_{i}}(t) \cdot \lambda_{2}^{1 / q_{i}}(t) \mathrm{d} t\right) \\
& \leqslant\|Y\|_{\mathbb{K}_{1, i}^{m}}\left(\int_{0}^{T} \mathbb{E}\left(\lambda_{i}(t)\right) \mathrm{d} t\right)^{1 / q_{i}} .
\end{aligned}
$$

(iii). It follows from

$$
X(\cdot) \cdot \lambda_{i}^{\frac{1}{q_{i}-1}}(\cdot) \in L^{q_{i}}\left(\mathfrak{M}^{m}, \lambda_{i}^{-\frac{1}{q_{i}-1}}(t, \omega) \cdot \mu^{m}(\mathrm{~d} t \times \mathrm{d} \omega) ; \mathbb{X}_{i}\right) \subset\left(\mathbb{K}_{1, i}^{m}\right)^{*} .
$$

(iv). The first conclusion follows from

$$
Y(\cdot) \cdot \lambda_{i}^{-1}(\cdot) \in L^{\frac{q_{i}}{q_{i}-1}}\left(\mathfrak{M}^{m}, \lambda_{i}(t, \omega) \cdot \mu^{m}(\mathrm{~d} t \times \mathrm{d} \omega) ; \mathbb{X}_{i}^{*}\right) \subset\left(\mathbb{K}_{2, i}^{m}\right)^{*}
$$

As for the second conclusion, by the well known Banach-Saks-Kakutani theorem, there exists a subsequence of $X_{n}$ (still denoted by $X_{n}$ ) such that its Césaro means $\tilde{X}_{n}$ strongly converges to $X$ and $\bar{X}$ in $\mathbb{K}_{2, i}^{m}$ and $\mathbb{K}_{5}^{m}$ respectively. Therefore, there is a subsequence $\tilde{X}_{n_{k}}$ such that for $\mu^{m}$-almost all $(t, \omega), \tilde{X}_{n_{k}}(t, \omega) \rightarrow X(t, \omega)$ in $\mathbb{X}$, and $\tilde{X}_{n_{k}}(t, \omega) \rightarrow \bar{X}(t, \omega)$ in $\mathbb{H}$. Since $\mathbb{X}$ is continuously and densely embedded in $\mathbb{H}$, we have $\bar{X}(t, \omega)=X(t, \omega)$ for $\mu^{m}$-almost all $(t, \omega)$.

(v). It follows from

$$
\begin{aligned}
\|J(G)\|_{\mathbb{K}_{4}^{m}}^{2} & =\mathbb{E}\left(\int_{0}^{\theta_{m}}\left\|\int_{0}^{t \wedge \theta_{m}} G(s) \mathrm{d} W(s)\right\|_{\mathbb{H}}^{2} \mathrm{~d} t\right) \\
& \leqslant \int_{0}^{T} \mathbb{E}\left(\int_{0}^{t \wedge \theta_{m}}\|G(s)\|_{L_{2}(\mathbb{U}, \mathbb{H})}^{2} \mathrm{~d} s\right) \mathrm{d} t \\
& \leqslant T\|G\|_{\mathbb{K}_{3}^{m}}^{2} .
\end{aligned}
$$

The proof is complete.

Definition 3.3. An $\mathbb{H}$-valued continuous $\mathcal{F}_{t}$-adapted process $X(t, \omega)$ is called a solution of Eq. (11) if for almost all $\omega \in \Omega$,

$$
X(\cdot, \omega) \in \cap_{i=1,2} L_{9}^{q_{i}}\left([0, T], \lambda_{i}(\cdot, \omega) \mathrm{d} t ; \mathbb{X}_{i}\right)
$$


and for all $t \in[0, T]$

$$
X(t, \omega)=X_{0}(\omega)+\int_{0}^{t} A(s, \omega, X(s, \omega)) \mathrm{d} s+\int_{0}^{t} B(s, X(s)) \mathrm{d} W(s)(\omega),
$$

where the first integral is understood as an $\mathbb{X}^{*}$-valued Bochner integral.

Remark 3.4. Note that

$$
\int_{0}^{t} A(s, \omega, X(s, \omega)) \mathrm{d} s=\int_{0}^{t} A_{1}(s, \omega, X(s, \omega)) \mathrm{d} s+\int_{0}^{t} A_{2}(s, \omega, X(s, \omega)) \mathrm{d} s .
$$

Since $X$ is $\mathcal{M} / \mathcal{B}(\mathbb{H})$-measurable, $1_{\mathbb{X}_{i}}(X) \cdot X$ is $\mathcal{M} / \mathcal{B}\left(\mathbb{X}_{i}\right)$-measurable by [16, Lemma 2.1] for $i=1,2$. The above integrals are meaningful.

We have the following estimates for the solutions of Eq.(11).

Theorem 3.5. Assume that $(\mathbf{H 1})-(\mathbf{H} 4)$ hold and $X_{0} \in L^{2}\left(\Omega, \mathcal{F}_{0}, P ; \mathbb{H}\right)$. Let $X(t)$ be any solution of Eq. (11) in the sense of Definition 3.3. Then, we have for any $m \in \mathbb{N}$

$$
\mathbb{E}\left\|X\left(\theta_{m}\right)\right\|_{\mathbb{H}}^{2}+\sum_{i=1,2}\|X\|_{\mathbb{K}_{2, i}^{m}}^{q_{i}}+\|X\|_{\mathbb{K}_{5}^{m}}^{2} \leqslant c_{m}\left(\mathbb{E}\left\|X_{0}\right\|_{\mathbb{H}}^{2}+\int_{0}^{T} \mathbb{E}(\xi(s)) \mathrm{d} s\right),
$$

and

$$
\begin{aligned}
& \mathbb{E}\left(\sup _{t \in\left[0, \theta_{m}\right]}\|X(t)\|_{\mathbb{H}}^{2}\right)+\|X\|_{\mathbb{K}_{4}^{m}}^{2}+\|B(\cdot, X(\cdot))\|_{\mathbb{K}_{3}^{m}}^{2}+\sum_{i=1,2}\left\|A_{i}(\cdot, X(\cdot))\right\|_{\mathbb{K}_{1, i}^{m}}^{\frac{q_{i}}{q_{i}}} \\
\leqslant & c_{m}\left(\mathbb{E}\left\|X_{0}\right\|_{\mathbb{H}}^{2}+\int_{0}^{T} \mathbb{E}\left(\xi(s)+\eta_{1}^{\frac{q_{1}}{q_{1}-1}}(s)+\eta_{2}^{\frac{q_{2}}{q_{2}-1}}(s)\right) \mathrm{d} s\right),
\end{aligned}
$$

where $c_{m}$ only depends on $m, T$ and $c_{A_{i}}, q_{i}, i=1,2$.

Proof. By Itô's formula (Theorem 2.1) and (H3), we have

$$
\begin{aligned}
& \|X(t)\|_{\mathbb{H}}^{2}-\left\|X_{0}\right\|_{\mathbb{H}}^{2}-M(t) \\
= & \int_{0}^{t}\left(2[X(s), A(s, X(s))]_{\mathbb{X}}+\|B(s, X(s))\|_{L_{2}(\mathbb{U}, \mathbb{H})}^{2}\right) \mathrm{d} s \\
\leqslant & \int_{0}^{t}\left(-\sum_{i=1,2}\left(\lambda_{i}(s) \cdot\|X(s)\|_{\mathbb{X}_{i}}^{q_{i}}\right)+\lambda_{3}(s) \cdot\|X(s)\|_{\mathbb{H}}^{2}+\xi(s)\right) \mathrm{d} s,
\end{aligned}
$$

where $M(t)$ is a continuous local martingale given by

$$
M(t):=2 \int_{0}^{t}\langle X(s), B(s, X(s)) \mathrm{d} W(s)\rangle_{\mathbb{H}} \cdot
$$

For any $R>0$, define the stopping time

$$
\tau_{R}:=\inf \left\{t \in[0, T]:\|X(t)\|_{\mathbb{H}} \geqslant R, \int_{0}^{t} \lambda_{i}(s) \cdot\|X(s)\|_{\mathbb{X}_{i}}^{q_{i}} \mathrm{~d} s \geqslant R, i=1,2\right\} .
$$

Then, by Definition $3.3, \tau_{R} \uparrow T$ a.s. as $R \uparrow \infty$.

By Remark 2.4 and the change of clock(cf. [25]), we know that $\left\{M\left(\theta_{t} \wedge \tau_{R}\right), t \geqslant 0\right\}$ is a continuous $\mathcal{F}_{\theta_{t}}$-martingale. Indeed, this follows from

$$
\left\langle M\left(\theta . \wedge \tau_{R}\right)\right\rangle(t) \leqslant 4 \int_{0}^{\theta_{t} \wedge \tau_{R}}\|X(s)\|_{\mathbb{H}}^{2} \cdot\|B(s, X(s))\|_{L_{2}(\mathbb{U}, \mathbb{H})}^{2} \mathrm{~d} s \leqslant c_{R} .
$$


So, replacing $t$ by $\theta_{t} \wedge \tau_{R}$ in (14) and taking expectations for both sides of (14), we obtain

$$
\begin{aligned}
& \mathbb{E}\left\|X\left(\theta_{t} \wedge \tau_{R}\right)\right\|_{\mathbb{H}}^{2}-\mathbb{E}\left\|X_{0}\right\|_{\mathbb{H}}^{2}+\sum_{i=1,2} \mathbb{E}\left(\int_{0}^{\theta_{t} \wedge \tau_{R}} \lambda_{i}(s) \cdot\|X(s)\|_{\mathbb{X}_{i}}^{q_{i}} \mathrm{~d} s\right) \\
\leqslant & \mathbb{E}\left(\int_{0}^{\theta_{t} \wedge \tau_{R}}\left(\lambda_{3}(s) \cdot\|X(s)\|_{\mathbb{H}}^{2}+\xi(s)\right) \mathrm{d} s\right) \\
= & \mathbb{E}\left(\int_{0}^{\theta_{t} \wedge \tau_{R}}\|X(s)\|_{\mathbb{H}}^{2} \mathrm{~d} H(s)\right)+\mathbb{E}\left(\int_{0}^{\theta_{t} \wedge \tau_{R}} \xi(s) \mathrm{d} s\right) \\
\leqslant & \mathbb{E}\left(\int_{0}^{\theta_{t}}\left\|X\left(s \wedge \tau_{R}\right)\right\|_{\mathbb{H}}^{2} \mathrm{~d} H(s)\right)+\int_{0}^{T} \mathbb{E}(\xi(s)) \mathrm{d} s \\
= & \int_{0}^{t} \mathbb{E}\left\|X\left(\theta_{s} \wedge \tau_{R}\right)\right\|_{\mathbb{H}}^{2} \mathrm{~d} s+\int_{0}^{T} \mathbb{E}(\xi(s)) \mathrm{d} s,
\end{aligned}
$$

where $H(s)$ is defined by (12), and in the last step we have used the variable substitution formula.

Hence, by Gronwall's inequality we have for any $t \geqslant 0$

$$
\mathbb{E}\left\|X\left(\theta_{t} \wedge \tau_{R}\right)\right\|_{\mathbb{H}}^{2} \leqslant e^{t}\left(\mathbb{E}\left\|X_{0}\right\|_{\mathbb{H}}^{2}+\int_{0}^{T} \mathbb{E}(\xi(s)) \mathrm{d} s\right)
$$

Letting $R \rightarrow \infty$, by Fatou's lemma we obtain that for any $m \in \mathbb{N}$

$$
\mathbb{E}\left\|X\left(\theta_{m}\right)\right\|_{\mathbb{H}}^{2} \leqslant e^{m}\left(\mathbb{E}\left\|X_{0}\right\|_{\mathbb{H}}^{2}+\int_{0}^{T} \mathbb{E}(\xi(s)) \mathrm{d} s\right)
$$

as well as

$$
\begin{aligned}
& \sum_{i=1,2} \mathbb{E}\left(\int_{0}^{\theta_{m}} \lambda_{i}(s) \cdot\|X(s)\|_{\mathbb{X}_{i}}^{q_{i}} \mathrm{~d} s\right)+\mathbb{E}\left(\int_{0}^{\theta_{m}} \lambda_{3}(s) \cdot\|X(s)\|_{\mathbb{H}}^{2} \mathrm{~d} s\right) \\
\leqslant & c_{m}\left(\mathbb{E}\left\|X_{0}\right\|_{\mathbb{H}}^{2}+\int_{0}^{T} \mathbb{E}(\xi(s)) \mathrm{d} s\right),
\end{aligned}
$$

which gives the first estimate.

From (14), by Burkholder's inequality and Young's inequality (5) we further have

$$
\begin{aligned}
& \mathbb{E}\left(\sup _{t \in\left[0, \theta_{m}\right]}\|X(t)\|_{\mathbb{H}}^{2}\right)-\mathbb{E}\left\|X_{0}\right\|_{\mathbb{H}}^{2} \\
\leqslant & \mathbb{E}\left(\int_{0}^{\theta_{m}}\left(\lambda_{3}(s) \cdot\|X(s)\|_{\mathbb{H}}^{2}+\xi(s)\right) \mathrm{d} s\right) \\
& +c_{0} \mathbb{E}\left(\int_{0}^{\theta_{m}}\|X(s)\|_{\mathbb{H}}^{2} \cdot\|B(s, X(s))\|_{L_{2}(\mathbb{U}, \mathbb{H})}^{2} \mathrm{~d} s\right)^{1 / 2} \\
\leqslant & \int_{0}^{T} \mathbb{E}(\xi(s)) \mathrm{d} s+\mathbb{E}\left(\int_{0}^{\theta_{m}} \lambda_{3}(s) \cdot\|X(s)\|_{\mathbb{H}}^{2} \mathrm{~d} s\right) \\
& +\frac{1}{2} \mathbb{E}\left(\sup _{t \in\left[0, \theta_{m}\right]}\|X(t)\|_{\mathbb{H}}^{2}\right)+c_{0} \mathbb{E}\left(\int_{0}^{\theta_{m}}\|B(s, X(s))\|_{L_{2}(\mathbb{U}, \mathbb{H})}^{2} \mathrm{~d} s\right) .
\end{aligned}
$$


Hence

$$
\begin{aligned}
\mathbb{E}\left(\sup _{t \in\left[0, \theta_{m}\right]}\|X(t)\|_{\mathbb{H}}^{2}\right) \leqslant & c_{m}\left(\mathbb{E}\left\|X_{0}\right\|_{\mathbb{H}}^{2}+\int_{0}^{T} \mathbb{E}(\xi(s)) \mathrm{d} s\right) \\
& +c_{0} \mathbb{E}\left(\int_{0}^{\theta_{m}}\|B(s, X(s))\|_{L_{2}(\mathbb{U}, \mathbb{H})}^{2} \mathrm{~d} s\right) .
\end{aligned}
$$

The second estimate now follows from (H4), Remark 2.4 and (16).

We now prove our main result in this section.

Theorem 3.6. Assume that $(A, B)$ satisfies $\mathscr{H}\left(\lambda_{0}, \lambda_{1}, \lambda_{2}, \lambda_{3}, \xi, \eta_{1}, \eta_{2}, q_{1}, q_{2}\right)$. Then for any $X_{0} \in L^{2}\left(\Omega, \mathcal{F}_{0}, P ; \mathbb{H}\right)$, there exists a unique solution to Eq.(11) in the sense of Definition 3.3.

Proof. (Uniqueness)

Let $X_{1}$ and $X_{2}$ be two solutions of Eq.(11) in the sense of Definition 3.3. For $t \geqslant 0$, define

$$
\beta_{t}:=\inf \left\{s \in[0, T]: \int_{0}^{s} \lambda_{0}(r) \mathrm{d} r \geqslant t\right\},
$$

and for $R>0$ and $i=1,2$, let $\tau_{R}^{i}$ be defined as in (15) corresponding to $X_{i}$. For $t_{0} \in(0, T)$, set $\tau_{R}^{t_{0}}:=\tau_{R}^{1} \wedge \tau_{R}^{2} \wedge t_{0}$. By Itô's formula(Theorem [2.1), as in the proof of Theorem 3.5 we have

$$
\begin{aligned}
& \mathbb{E}\left\|\left(X_{1}-X_{2}\right)\left(\beta_{t} \wedge \tau_{R}^{t_{0}}\right)\right\|_{\mathbb{H}}^{2} \\
= & \mathbb{E}\left(\int _ { 0 } ^ { \beta _ { t } \wedge \tau _ { R } ^ { t _ { 0 } } } \left(2\left[X_{1}(s)-X_{2}(s), A\left(s, X_{1}(s)\right)-A\left(s, X_{2}(s)\right)\right]_{\mathbb{X}}\right.\right. \\
& \left.\left.+\left\|B\left(s, X_{1}(s)\right)-B\left(s, X_{2}(s)\right)\right\|_{L_{2}(\mathbb{U}, \mathbb{H})}^{2}\right) \mathrm{~d} s\right) \\
\leqslant & \mathbb{E}\left(\int_{0}^{\beta_{t} \wedge \tau_{R}^{t_{0}}}\left\|X_{1}(s)-X_{2}(s)\right\|_{\mathbb{H}}^{2} \cdot \lambda_{0}(s) \mathrm{d} s\right) \\
\leqslant & \mathbb{E}\left(\int_{0}^{t}\left\|\left(X_{1}-X_{2}\right)\left(\beta_{s} \wedge \tau_{R}^{t_{0}}\right)\right\|_{\mathbb{H}}^{2} \mathrm{~d} s\right) .
\end{aligned}
$$

Using Gronwall's inequality yields that for any $t \geqslant 0$ and $R>0$

$$
\mathbb{E}\left\|\left(X_{1}-X_{2}\right)\left(\beta_{t} \wedge \tau_{R}^{t_{0}}\right)\right\|_{\mathbb{H}}^{2}=0 .
$$

Letting $R, t \rightarrow \infty$, and by Fatou's lemma we get

$$
\mathbb{E}\left\|\left(X_{1}-X_{2}\right)\left(t_{0}\right)\right\|_{\mathbb{H}}^{2}=0 .
$$

The uniqueness is then obtained.

(Existence) We divide the proof into five steps.

(Step 1)

First of all, let us reduce (H1)-(H4) to the case of $\lambda_{0}=0$. Let $X$ be a solution of Eq.(11). Set

$$
\begin{aligned}
\gamma(t, \omega) & :=e^{\frac{1}{2} \int_{0}^{t} \lambda_{0}(s, \omega) \mathrm{d} s} \\
\tilde{X}(t, \omega) & :=\gamma^{-1}(t, \omega) \cdot X(t, \omega)
\end{aligned}
$$




$$
\begin{aligned}
\tilde{A}_{i}(t, \omega, x) & :=\gamma^{-1}(t, \omega) \cdot A_{i}(t, \omega, \gamma(t, \omega) x)-\frac{\lambda_{0}(t, \omega) x}{2}, \quad i=1,2 \\
\tilde{B}(t, \omega, x) & :=\gamma^{-1}(t, \omega) \cdot B(t, \omega, \gamma(t, \omega) x)
\end{aligned}
$$

Noticing that $\gamma^{-1}(t) \leqslant 1$, we have

$$
\begin{aligned}
& 2[x, \tilde{A}(t, x)]_{\mathbb{X}}+\|\tilde{B}(t, x)\|_{L_{2}(\mathbb{U}, \mathbb{H})}^{2} \\
\leqslant & \gamma^{-2}(t)\left(-\sum_{i=1,2} \lambda_{i}(t) \cdot \gamma^{q_{i}}(t) \cdot\|x\|_{\mathbb{X}_{i}}^{q_{i}}+\lambda_{3}(t) \cdot \gamma^{2}(t) \cdot\|x\|_{\mathbb{H}}^{2}+\xi(t)\right)+\lambda_{0}(t) \cdot\|x\|_{\mathbb{H}}^{2} \\
\leqslant & -\sum_{i=1,2}\left(\lambda_{i}(t) \cdot \gamma^{q_{i}-2}(t) \cdot\|x\|_{\mathbb{X}_{i}}^{q_{i}}\right)+\left(\lambda_{3}(t)+\lambda_{0}(t)\right) \cdot\|x\|_{\mathbb{H}}^{2}+\xi(t),
\end{aligned}
$$

On the other hand, by ([6) and Young's inequality (5) and $q_{1}, q_{2} \geqslant 2$, we have for $i=1,2$

$$
\begin{aligned}
\left\|\tilde{A}_{i}(t, x)\right\|_{\mathbb{X}_{i}^{*}} & \leqslant \gamma^{-1}(t) \cdot\left\|A_{i}(t, \gamma(t) x)\right\|_{\mathbb{X}_{i}^{*}}+\frac{\lambda_{0}(t) \cdot\|x\|_{\mathbb{X}_{i}^{*}}}{2} \\
& \leqslant \gamma^{-1}(t)\left(\eta_{i}(t) \cdot \lambda_{i}^{1 / q_{i}}(t)+c_{A_{i}} \gamma^{q_{i}-1}(t) \cdot \lambda_{i}(t) \cdot\|x\|_{\mathbb{X}_{i}}^{q_{i}-1}\right)+c_{0} \lambda_{i}(t) \cdot\|x\|_{\mathbb{X}_{i}} \\
& \leqslant\left(\eta_{i}(t)+c_{0} \lambda_{i}^{\frac{q_{i}-1}{q_{i}}}(t)\right) \lambda_{i}^{1 / q_{i}}(t)+\left(c_{A_{i}} \gamma^{q_{i}-2}(t)+c_{0}\right) \lambda_{i}(t) \cdot\|x\|_{\mathbb{X}_{i}}^{q_{i}-1} \\
& \leqslant\left(\eta_{i}(t)+c_{0} \lambda_{i}^{\frac{q_{i}-1}{q_{i}}}(t)\right)\left(\gamma^{q_{i}-2}(t) \cdot \lambda_{i}(t)\right)^{1 / q_{i}}+c_{0} \gamma^{q_{i}-2}(t) \cdot \lambda_{i}(t) \cdot\|x\|_{\mathbb{X}_{i}}^{q_{i}-1} .
\end{aligned}
$$

Thus, $(\tilde{A}, \tilde{B})$ satisfies $\mathscr{H}\left(0, \tilde{\lambda}_{1}, \tilde{\lambda}_{2}, \lambda_{3}+\lambda_{0}, \xi, \tilde{\eta}_{1}, \tilde{\eta}_{2}, q_{1}, q_{2}\right)$ with

$$
\begin{aligned}
\tilde{\lambda}_{i}(t) & :=\lambda_{i}(t) \cdot e^{\frac{q_{i}-2}{2} \int_{0}^{t} \lambda_{0}(s) \mathrm{d} s} \in L^{1}(\mathfrak{A}), \quad i=1,2, \\
\tilde{\eta}_{i}(t) & :=\eta_{i}(t)+c_{0} \lambda_{i}^{\frac{q_{i}-1}{q_{i}}}(t) \in L^{\frac{q_{i}}{q_{i}-1}}(\mathfrak{A}), \quad i=1,2 .
\end{aligned}
$$

Moreover, it holds that

$$
\mathrm{d} \tilde{X}(t)=\tilde{A}(t, \tilde{X}(t)) \mathrm{d} t+\tilde{B}(t, \tilde{X}(t)) \mathrm{d} W(t), \quad \tilde{X}(0)=X(0) .
$$

It is easy to see that

$$
X(\cdot, \omega) \in \cap_{i=1,2} L^{q_{i}}\left([0, T], \lambda_{i}(\cdot, \omega) \mathrm{d} t ; \mathbb{X}_{i}\right)
$$

is equivalent to

$$
\tilde{X}(\cdot, \omega) \in \cap_{i=1,2} L^{q_{i}}\left([0, T], \tilde{\lambda}_{i}(\cdot, \omega) \mathrm{d} t ; \mathbb{X}_{i}\right) .
$$

So, we may assume that $\lambda_{0}=0$ in the following proof.

(Step 2)

We now use Galerkin's approximation to prove the existence of solutions.

Let $\left\{e_{i}, i \in \mathbb{N}\right\} \subset \mathbb{X}$ be a normal orthogonal basis of $\mathbb{H}$. Set

$$
\Pi_{n} x:=\sum_{i=1}^{n}\left[e_{i}, x\right]_{\mathbb{X}} \cdot e_{i}, \quad x \in \mathbb{X}^{*}
$$

Then, the mapping $\Pi_{n}: \mathbb{X}^{*} \mapsto \mathbb{X}$ is linear and continuous, and satisfy

$$
\Pi_{n} x=\sum_{i=1}^{n}\left\langle e_{i}, x\right\rangle_{\mathbb{H}} \cdot e_{i}, \quad x \in \mathbb{H}
$$

and

$$
\left[\Pi_{n} x, y\right]_{\mathbb{X}}=\left[\Pi_{n} y, x\right]_{\mathbb{X}}, \quad x, y \in \mathbb{X}^{*}
$$


We also fix a normal orthogonal basis $\left\{f_{1}, f_{2}, \cdots\right\}$ of $\mathbb{U}$. Let $W_{j}(t):=\left\langle W(t), f_{j}\right\rangle_{\mathbb{U}}$ for $j \in \mathbb{N}$. Consider the following Itô type stochastic ordinary differential equation in $\mathbb{R}^{n}$ :

$$
\left\{\begin{array}{l}
\mathrm{d} X_{n}^{i}(t)=b^{i}\left(t, X_{n}(t)\right) \mathrm{d} t+\sum_{j=1}^{n} \sigma_{j}^{i}\left(t, X_{n}(t)\right) \mathrm{d} W_{j}(t), \\
X_{n}^{i}(0)=\left\langle X_{0}, e_{i}\right\rangle_{\mathbb{H}}, \quad i=1, \cdots, n,
\end{array}\right.
$$

where $b^{i}(t, x):=\left[e_{i}, A(t, x \cdot e)\right]_{\mathbb{X}}$, and $\sigma_{j}^{i}(t, x):=\left\langle e_{i}, B(t, x \cdot e)\left(f_{j}\right)\right\rangle_{\mathbb{H}}$. Here, $x \in \mathbb{R}^{n}$ and $x \cdot e:=\sum_{i=1}^{n} x^{i} e_{i}$.

The coefficients satisfy the following conditions by $(\mathbf{H 1})-(\mathbf{H} 4)$ :

(i) $b$ and $\sigma$ are $\mathcal{M} \times \mathcal{B}\left(\mathbb{R}^{n}\right) / \mathcal{B}\left(\mathbb{R}^{n}\right)$ and $\mathcal{M} \times \mathcal{B}\left(\mathbb{R}^{n}\right) / \mathcal{B}\left(L_{2}\left(\mathbb{R}^{n}, \mathbb{R}^{n}\right)\right)$-measurable respectively and continuous in $x$.

(ii) For any $(t, \omega) \in[0, T] \times \Omega$ and $x, y \in \mathbb{R}^{n}$

$$
2\langle x-y, b(t, \omega, x)-b(t, \omega, y)\rangle_{\mathbb{R}^{n}}+\|\sigma(t, \omega, x)-\sigma(t, \omega, y)\|_{L_{2}\left(\mathbb{R}^{n}, \mathbb{R}^{n}\right)}^{2} \leqslant 0 .
$$

(iii) For any $(t, \omega) \in[0, T] \times \Omega$ and $x \in \mathbb{R}^{n}$

$$
2\langle x, b(t, \omega, x)\rangle_{\mathbb{R}^{n}}+\|\sigma(t, \omega, x)\|_{L_{2}\left(\mathbb{R}^{n}, \mathbb{R}^{n}\right)}^{2} \leqslant \lambda_{3}(t, \omega) \cdot\|x\|_{\mathbb{R}^{n}}^{2}+\xi(t, \omega) .
$$

(iv) For any $(t, \omega) \in[0, T] \times \Omega$ and $x \in \mathbb{R}^{n}$

$$
\|b(t, \omega, x)\|_{\mathbb{R}^{n}} \leqslant c_{n} \sum_{i=1,2}\left(\eta_{i}(t, \omega) \cdot \lambda_{i}^{1 / q_{i}}(t, \omega)+\lambda_{i}(t, \omega) \cdot\|x\|_{\mathbb{R}^{n}}^{q_{i}-1}\right) .
$$

By the well-known result (cf. [14]), there exists a unique continuous $\mathcal{F}_{t}$-adapted solution denoted by $X_{n}^{i}(t)$ to Eq.(17). Moreover, if we let $X_{n}(t):=\sum_{i=1}^{n} X_{n}^{i}(t) e_{i}$, then we can write Eq.(17) as

$$
X_{n}(t)=\Pi_{n} X_{0}+\int_{0}^{t} \Pi_{n} A\left(s, X_{n}(s)\right) \mathrm{d} s+\int_{0}^{t} \Pi_{n} B\left(s, X_{n}(s)\right) \tilde{\Pi}_{n} \mathrm{~d} W(s),
$$

where $\tilde{\Pi}_{n}$ is the projection on $\operatorname{span}\left\{f_{1}, \cdots, f_{n}\right\}$ in $\mathbb{U}$.

Noticing that

$$
\left\|\Pi_{n} B\left(s, X_{n}(s)\right) \tilde{\Pi}_{n}\right\|_{L_{2}(\mathbb{U}, \mathbb{H})} \leqslant\left\|B\left(s, X_{n}(s)\right)\right\|_{L_{2}(\mathbb{U}, \mathbb{H})},
$$

and using the same method as in the proof of Theorem 3.5, by $(\mathbf{H 4})$ and Remark 2.4, we have for all $n \in \mathbb{N}$

$$
\begin{aligned}
& \mathbb{E}\left\|X_{n}\left(\theta_{m}\right)\right\|_{\mathbb{H}}^{2}+\left\|X_{n}\right\|_{\mathbb{K}_{4}^{m}}^{2}+\left\|X_{n}\right\|_{\mathbb{K}_{5}^{m}}^{2}+\left\|B\left(\cdot, X_{n}\right)\right\|_{\mathbb{K}_{3}^{m}}^{2} \\
& +\sum_{i=1,2}\left(\left\|X_{n}\right\|_{\mathbb{K}_{2, i}^{m}}^{q_{i}}+\left\|A_{i}\left(\cdot, X_{n}\right)\right\|_{\mathbb{K}_{i}^{m}}^{\frac{q_{i}}{q_{i}-1}}\right) \\
\leqslant & c_{m}\left(\mathbb{E}\left\|X_{0}\right\|_{\mathbb{H}}^{2}+\int_{0}^{T} \mathbb{E}\left(\xi(s)+\eta_{1}^{\frac{q_{1}}{q_{1}-1}}(s)+\eta_{2}^{\frac{q_{2}}{q_{2}-1}}(s)\right) \mathrm{d} s\right)<+\infty,
\end{aligned}
$$

where $c_{m}>0$ is independent of $n$, and $m \in \mathbb{N}$ is fixed in the next two steps.

(Step 3)

By the reflexivities of Banach spaces $\mathbb{K}^{m}$, one may find a common subsequence $n_{k}$ (denoted by $k$ for simplicity) and $\tilde{X}^{m} \in \mathbb{K}_{2,1}^{m} \cap \mathbb{K}_{2,2}^{m}, \bar{X}^{m} \in \mathbb{K}_{4}^{m} \cap \mathbb{K}_{5}^{m}, Y_{i}^{m} \in \mathbb{K}_{1, i}^{m}, i=1,2$, $Z^{m} \in \mathbb{K}_{3}^{m}$ and $X_{\infty}^{m} \in L^{2}\left(\Omega, \mathcal{F}_{\theta_{m}}, P ; \mathbb{H}\right)$ such that as $k \rightarrow \infty$

$$
\begin{aligned}
X_{k} & \rightarrow \tilde{X}^{m} \text { weakly in } \mathbb{K}_{2,1}^{m} \text { and } \mathbb{K}_{2,2}^{m}, \\
X_{k} & \rightarrow \bar{X}^{m} \text { weakly in } \mathbb{K}_{4}^{m} \text { and } \mathbb{K}_{5}^{m}, \\
A_{i}\left(\cdot, X_{k}\right)=: Y_{k, i} & \rightarrow Y_{i}^{m} \text { weakly in } \mathbb{K}_{1, i}^{m}, \quad i=1,2, \\
B\left(\cdot, X_{k}\right) \tilde{\Pi}_{k}=: Z_{k} & \rightarrow Z^{m} \text { weakly in } \mathbb{K}_{3}^{m}, \\
&
\end{aligned}
$$




$$
X_{k}\left(\theta_{m}\right) \rightarrow X_{\infty}^{m} \text { weakly in } L^{2}\left(\Omega, \mathcal{F}_{\theta_{m}}, P ; \mathbb{H}\right) .
$$

Clearly, $\tilde{X}^{m}, \bar{X}^{m}, Y_{1}^{m}, Y_{2}^{m}$ and $Z^{m}$ are $\mathcal{M}$-measurable. First of all, by (iv) of Lemma 3.2, we have

$$
\tilde{X}^{m}(t, \omega)=\bar{X}^{m}(t, \omega) \text {, for } \mu^{m} \text {-almost all }(t, \omega) .
$$

Secondly, define

$$
X^{m}(t):=X_{0}+\int_{0}^{t \wedge \theta_{m}}\left(Y_{1}^{m}(s)+Y_{2}^{m}(s)\right) \mathrm{d} s+\int_{0}^{t \wedge \theta_{m}} Z^{m}(s) \mathrm{d} W(s),
$$

then $t \mapsto X^{m}(t, \omega)$ is continuous in $\mathbb{H}$ a.s. and (cf. [27])

$$
\mathbb{E}\left(\sup _{t \in\left[0, \theta_{m}\right]}\left\|X^{m}(t)\right\|_{\mathbb{H}}^{2}\right)<+\infty .
$$

Moreover, we also have

$$
X^{m}(t, \omega)=\bar{X}^{m}(t, \omega) \quad \text { for } \mu^{m} \text {-almost all }(t, \omega) .
$$

Indeed, let $\zeta(t)$ be any $\mathbb{H}$-valued bounded and measurable process on $(\Omega, \mathcal{F}, P)$. By (18) we have for any $k \geqslant n$

$$
\begin{aligned}
\mathbb{E}\left(\int_{0}^{\theta_{m}}\left\langle\Pi_{n} \zeta(t), X_{k}(t)\right\rangle_{\mathbb{H}} \mathrm{d} t\right)= & \mathbb{E}\left(\int_{0}^{\theta_{m}}\left\langle\Pi_{n} \zeta(t), \Pi_{k} X_{0}\right\rangle_{\mathbb{H}} \mathrm{d} t\right) \\
& +\mathbb{E}\left(\int_{0}^{\theta_{m}} \int_{0}^{t}\left[\Pi_{n} \zeta(t), Y_{k, 1}(s)+Y_{k, 2}(s)\right]_{\mathbb{X}} \mathrm{d} s \mathrm{~d} t\right) \\
& +\mathbb{E}\left(\int_{0}^{\theta_{m}}\left\langle\Pi_{n} \zeta(t), J\left(Z_{k}\right)(t)\right\rangle_{\mathbb{H}} \mathrm{d} t\right),
\end{aligned}
$$

where $J$ is defined by (13), and we have used that

$$
\left\langle\Pi_{n} \zeta(t), J\left(\Pi_{k} Z_{k}\right)(t)\right\rangle_{\mathbb{H}}=\left\langle\Pi_{n} \zeta(t), \Pi_{k} J\left(Z_{k}\right)(t)\right\rangle_{\mathbb{H}}=\left\langle\Pi_{n} \zeta(t), J\left(Z_{k}\right)(t)\right\rangle_{\mathbb{H}} .
$$

Taking limits for $k \rightarrow \infty$, and by Fubini's theorem, (20) (21) (22) and (iii), (v) of Lemma 3.2 we obtain

$$
\mathbb{E}\left(\int_{0}^{\theta_{m}}\left\langle\Pi_{n} \zeta(t), \bar{X}^{m}(t)\right\rangle_{\mathbb{H}} \mathrm{d} t\right)=\mathbb{E}\left(\int_{0}^{\theta_{m}}\left\langle\Pi_{n} \zeta(t), X^{m}(t)\right\rangle_{\mathbb{H}} \mathrm{d} t\right) .
$$

Letting $n \rightarrow \infty$ then shows (25) by the arbitrariness of $\zeta$. Using the same method, by (21) (22) and (23) we also have

$$
X^{m}\left(\theta_{m}(\omega), \omega\right)=X_{\infty}^{m}(\omega) \quad \text { for } P \text {-almost all } \omega \in \Omega .
$$

In the following we shall not distinguish $X^{m}, \tilde{X}^{m}$ and $\bar{X}^{m}$.

(Step 4)

Our task in this step is to show by the standard monotone argument that for $\mu^{m}$-almost all $(t, \omega) \in[0, T] \times \Omega$

$$
\begin{aligned}
\left(A_{1}+A_{2}\right)\left(t, \omega, X^{m}(t, \omega)\right) & =\left(Y_{1}^{m}+Y_{2}^{m}\right)(t, \omega)=: Y^{m}(t, \omega), \\
B\left(t, \omega, X^{m}(t, \omega)\right) & =Z^{m}(t, \omega) .
\end{aligned}
$$

By Ito's formula and (H2)(with $\lambda_{0}=0$ ), from (18) we have for any $\Phi \in \mathbb{K}_{2,1}^{m} \cap \mathbb{K}_{2,2}^{m} \cap \mathbb{K}_{5}^{m}$

$$
\begin{aligned}
& \left\|X_{k}\left(\theta_{m}\right)\right\|_{\mathbb{H}}^{2}-\left\|\Pi_{k} X_{0}\right\|_{\mathbb{H}}^{2}-2 M\left(\theta_{m}\right) \\
= & \int_{0}^{\theta_{m}}\left(2\left[X_{k}(s), A\left(s, X_{k}(s)\right)\right]_{\mathbb{X}}+\left\|\Pi_{k} B\left(s, X_{k}(s)\right) \tilde{\Pi}_{k}\right\|_{L_{2}(\mathbb{U}, \mathbb{H})}^{2}\right) \mathrm{d} s
\end{aligned}
$$




$$
\begin{aligned}
\leqslant & \int_{0}^{\theta_{m}}\left(2\left[X_{k}(s), A\left(s, X_{k}(s)\right)\right]_{\mathbb{X}}+\left\|B\left(s, X_{k}(s)\right)\right\|_{L_{2}(\mathbb{U}, \mathbb{H})}^{2}\right) \mathrm{d} s \\
\leqslant & \int_{0}^{\theta_{m}}\left(2\left[X_{k}(s), A(s, \Phi(s))\right]_{\mathbb{X}}+2\left[\Phi(s), A\left(s, X_{k}(s)\right)-A(s, \Phi(s))\right]_{\mathbb{X}}\right. \\
& \left.-\|B(s, \Phi(s))\|_{L_{2}(\mathbb{U}, \mathbb{H})}^{2}+2\left\langle B\left(s, X_{k}(s)\right), B(s, \Phi(s))\right\rangle_{L_{2}(\mathbb{U}, \mathbb{H})}\right) \mathrm{d} s,
\end{aligned}
$$

where $M(t)$ is a continuous martingale defined by

$$
M(t):=\int_{0}^{t \wedge \theta_{m}}\left\langle X_{k}(s), B\left(s, X_{k}(s)\right) \tilde{\Pi}_{k} \mathrm{~d} W(s)\right\rangle_{\mathbb{H} \cdot}
$$

Since $\Phi \in \mathbb{K}_{2,1}^{m} \cap \mathbb{K}_{2,2}^{m} \cap \mathbb{K}_{5}^{m}$, we have $B(\cdot, \Phi(\cdot)) \in \mathbb{K}_{3}^{m}$ by Remark 2.4. Firstly taking expectations for (27), and then taking limits for $k \rightarrow \infty$, we find by (19) (21) (22) and (iii) (iv) of Lemma 3.2 that

$$
\begin{aligned}
& \liminf _{k \rightarrow \infty} \mathbb{E}\left\|X_{k}\left(\theta_{m}\right)\right\|_{\mathbb{H}}^{2}-\mathbb{E}\left\|X_{0}\right\|_{\mathbb{H}}^{2} \\
\leqslant & \mathbb{E}\left(\int _ { 0 } ^ { \theta _ { m } } \left(2\left[X^{m}(s), A(s, \Phi(s))\right]_{\mathbb{X}}+2\left[\Phi(s), Y^{m}(s)-A(s, \Phi(s))\right]_{\mathbb{X}}\right.\right. \\
& \left.\left.-\|B(s, \Phi(s))\|_{L_{2}(\mathbb{U}, \mathbb{H})}^{2}+2\left\langle Z^{m}(s), B(s, \Phi(s))\right\rangle_{L_{2}(\mathbb{U}, \mathbb{H})}\right) \mathrm{d} s\right) .
\end{aligned}
$$

On the other hand, from (24), noting that by Ito's formula again

$$
\mathbb{E}\left\|X^{m}\left(\theta_{m}\right)\right\|_{\mathbb{H}}^{2}-\mathbb{E}\left\|X_{0}\right\|_{\mathbb{H}}^{2}=\mathbb{E}\left(\int_{0}^{\theta_{m}}\left(2\left[X^{m}(s), Y^{m}(s)\right]_{\mathbb{X}}+\left\|Z^{m}(s)\right\|_{L_{2}(\mathbb{U}, \mathbb{H})}^{2}\right) \mathrm{d} s\right)
$$

and by (23) and (26)

$$
\mathbb{E}\left\|X^{m}\left(\theta_{m}\right)\right\|_{\mathbb{H}}^{2} \leqslant \liminf _{k \rightarrow \infty} \mathbb{E}\left\|X_{k}\left(\theta_{m}\right)\right\|_{\mathbb{H}}^{2}
$$

we finally arrive at

$$
\begin{aligned}
& \mathbb{E}\left(\int_{0}^{\theta_{m}} 2\left[X^{m}(s)-\Phi(s), Y^{m}(s)-A(s, \Phi(s))\right]_{\mathbb{X}} \mathrm{d} s\right) \\
& +\mathbb{E}\left(\int_{0}^{\theta_{m}}\left\|B(s, \Phi(s))-Z^{m}(s)\right\|_{L_{2}(\mathbb{U}, \mathbb{H})}^{2} \mathrm{~d} s\right) \leqslant 0 .
\end{aligned}
$$

Letting $\Phi=X^{m}$ in the above inequality, we obtain that $Z^{m}=B\left(\cdot, X^{m}\right)$. By Lemma 2.5 we also have $Y^{m}=A\left(\cdot, X^{m}\right)$.

(Step 5)

For $m \geqslant l$, since $\theta_{m}(\omega) \geqslant \theta_{l}(\omega)$ a.s., both $X^{m}(\cdot, \omega)$ and $X^{l}(\cdot, \omega)$ solve the following equation

$$
\mathrm{d} X(t)=A(t, X(t)) 1_{\left\{t \leqslant \theta_{l}\right\}} \mathrm{d} t+B(t, X(t)) 1_{\left\{t \leqslant \theta_{l}\right\}} \mathrm{d} W(s), \quad X(0)=X_{0} .
$$

The uniqueness of solutions gives that for almost all $\omega$

$$
X^{m}(t, \omega)=X^{l}(t, \omega), \quad t \leqslant \theta_{l}(\omega) .
$$

Thus, noting that $\theta_{m}(\omega) \uparrow T$ a.s. as $m \uparrow \infty$, we may define a continuous $\mathcal{F}_{t}$-adapted $\mathbb{H}$-valued process for all $t \in(0, T)$ by

$$
X(t, \omega):=X^{m}(t, \omega) \quad \text { if } t<\theta_{m}(\omega),
$$

Clearly, it is a solution of Eq.(11) in the sense of Definition 3.3. The proof is complete. 
Remark 3.7. Since $q_{1}, q_{2} \geqslant 2$ is only used in Step 1, if $\lambda_{0}=0$, Theorem 3.6 still holds for any $q_{1}, q_{2}>1$.

\section{Backward Stochastic Evolution Equations}

In this section, we consider the following type of backward stochastic evolution equation:

$$
\left\{\begin{array}{l}
\mathrm{d} X(t)=-A(t, X(t)) \mathrm{d} t-C(t, X(t), Z(t)) \mathrm{d} t+Z(t) \mathrm{d} W(t) \\
X(T)=X_{T} \in \mathcal{F}_{T} / \mathcal{B}(\mathbb{H})
\end{array}\right.
$$

where

$$
\begin{aligned}
A_{i}:[0, T] \times \Omega \times \mathbb{X}_{i} \mapsto \mathbb{X}_{i}^{*} & \in \mathcal{M} \times \mathcal{B}\left(\mathbb{X}_{i}\right) / \mathcal{B}\left(\mathbb{X}_{i}^{*}\right), \quad i=1,2 \\
C:[0, T] \times \Omega \times \mathbb{H} \times L_{2}(\mathbb{U}, \mathbb{H}) & \mapsto \mathbb{H} \in \mathcal{M} \times \mathcal{B}(\mathbb{H}) \times \mathcal{B}\left(L_{2}(\mathbb{U}, \mathbb{H})\right) / \mathcal{B}(\mathbb{H})
\end{aligned}
$$

We assume that

(HB1) $X_{T} \in L^{2}\left(\Omega, \mathcal{F}_{T}, P ; \mathbb{H}\right)$ and $(A, 0)$ satisfies $\mathscr{H}\left(\lambda_{0}, \lambda_{1}, \lambda_{2}, \lambda_{3}, \xi, \eta_{1}, \eta_{2}, q_{1}, q_{2}\right)$, where $\lambda_{i}, i=0,1,2,3$ are positive constants, $q_{i} \geqslant 2,0<\xi \in L^{1}(\mathfrak{A})$ and

$$
\mathbb{E}\left(\int_{0}^{T}\left|\eta_{1}(s)+\eta_{2}(s)\right|^{2} \mathrm{~d} s\right)^{\left(q_{1} \vee q_{2}\right) / 2}<+\infty .
$$

(HB2) There exist a $c_{1}>0$ and an increasing concave function $\rho$ satisfying (3) such that for all $(t, \omega) \in[0, T] \times \Omega, x, x^{\prime} \in \mathbb{H}$ and $z, z^{\prime} \in L_{2}(\mathbb{U}, \mathbb{H})$

$$
\left\|C(t, \omega, x, z)-C\left(t, \omega, x^{\prime}, z^{\prime}\right)\right\|_{\mathbb{H}}^{2} \leqslant c_{1}\left(\rho\left(\left\|x-x^{\prime}\right\|_{\mathbb{H}}^{2}\right)+\left\|z-z^{\prime}\right\|_{L_{2}(\mathbb{U}, \mathbb{H})}^{2}\right) .
$$

(HB3) There exist a $c_{2}>0$ and a $0<\zeta \in L^{2}(\mathfrak{A})$ such that for all $(t, \omega) \in[0, T] \times \Omega$, $x \in \mathbb{H}$ and $z \in L_{2}(\mathbb{U}, \mathbb{H})$

$$
\|C(t, \omega, x, z)\|_{\mathbb{H}} \leqslant \zeta(t, \omega)+c_{2}\left(\|x\|_{\mathbb{H}}+\|z\|_{L_{2}(\mathbb{U}, \mathbb{H})}\right) .
$$

Recalling Remark 3.1, we give the following definition.

Definition 4.1. A pair of measurable $\mathcal{F}_{t}$-adapted processes $(X, Z)$ is called a solution of $E q .(28)$ if

(i) $X \in \mathbb{K}_{2,1} \cap \mathbb{K}_{2,2} \cap \mathbb{K}_{4}$ and $Z \in \mathbb{K}_{3}, X(0) \in L^{2}\left(\Omega, \mathcal{F}_{0}, P ; \mathbb{H}\right)$.

(ii) For almost all $\omega, t \mapsto X(t, \omega)$ is continuous in $\mathbb{H}$ and $X(T)=X_{T}$ a.s..

(iii) $(X, Z)$ satisfies that in $\mathbb{X}^{*}$, for almost all $\omega$ and all $t \in[0, T]$

$$
X(t)=X_{T}+\int_{t}^{T} A(s, X(s)) \mathrm{d} s+\int_{t}^{T} C(s, X(s), Z(s)) \mathrm{d} s-\int_{t}^{T} Z(s) \mathrm{d} W(s) .
$$

As in Step 1 of Theorem [3.6, let $\gamma(t):=e^{\lambda_{0} t / 2}$ and define

$$
\begin{aligned}
(\tilde{X}(t, \omega), \tilde{Z}(t, \omega)) & :=(\gamma(t) \cdot X(t, \omega), \gamma(t) \cdot Z(t, \omega)), \\
\tilde{A}_{i}(t, \omega, x) & :=\gamma(t) \cdot A_{i}\left(t, \omega, \gamma^{-1}(t) \cdot x\right)-\lambda_{0} \cdot x / 2, \quad i=1,2, \\
\tilde{C}(t, \omega, x, z) & :=\gamma(t) \cdot C\left(t, \omega, \gamma^{-1}(t) \cdot x, \gamma^{-1}(t) \cdot z\right) .
\end{aligned}
$$

Then we can assume that $\lambda_{0}=0$ in (HB1) in the following.

We have the following uniqueness result.

Theorem 4.2. Assume that (HB1), (HB2) and (HB3) hold. Let $(X, Z)$ and $(\tilde{X}, \tilde{Z})$ be two solutions of $E q$. (28) with the same terminal values $X_{T}$. Then for $(\mathrm{d} t \times \mathrm{d} P)$-almost all $(t, \omega) \in[0, T] \times \Omega$

$$
X(t, \omega)=\tilde{X}(t, \omega), \quad Z(t, \omega)=\tilde{Z}(t, \omega)
$$


Proof. Set $Y(t):=X(t)-\tilde{X}(t)$. By Itô's formula(Theorem 2.1), we have

$$
\begin{aligned}
& \|Y(t)\|_{\mathbb{H}}^{2}+\int_{t}^{T}\|Z(s)-\tilde{Z}(s)\|_{L_{2}(\mathbb{U}, \mathbb{H})}^{2} \mathrm{~d} s \\
= & 2 \int_{t}^{T}[Y(s), A(s, X(s))-A(s, \tilde{X}(s))]_{\mathbb{X}} \mathrm{d} s \\
& +2 \int_{t}^{T}\langle Y(s), C(s, X(s), Z(s))-C(s, \tilde{X}(s), \tilde{Z}(s))\rangle_{\mathbb{H}} \mathrm{d} s \\
& -2 \int_{t}^{T}\langle Y(s),(Z(s)-\tilde{Z}(s)) \mathrm{d} W(s)\rangle_{\mathbb{H}} .
\end{aligned}
$$

Taking expectations, by $(\mathbf{H 2})\left(\right.$ with $\left.\lambda_{0}=0\right),($ HB2) and Young's inequality (5) we have

$$
\begin{aligned}
& \mathbb{E}\|Y(t)\|_{\mathbb{H}}^{2}+\int_{t}^{T} \mathbb{E}\|Z(s)-\tilde{Z}(s)\|_{L_{2}(\mathbb{U}, \mathbb{H})}^{2} \mathrm{~d} s \\
\leqslant & c_{0} \mathbb{E}\left(\int_{t}^{T}\|Y(s)\|_{\mathbb{H}}\left(\rho\left(\|Y(s)\|_{\mathbb{H}}^{2}\right)+\|Z(s)-\tilde{Z}(s)\|_{L_{2}(\mathbb{U}, \mathbb{H})}^{2}\right)^{1 / 2} \mathrm{~d} s\right) \\
\leqslant & c_{0} \int_{t}^{T} \mathbb{E}\|Y(s)\|_{\mathbb{H}}^{2} \mathrm{~d} s+\frac{1}{2} \int_{t}^{T} \mathbb{E} \rho\left(\|Y(s)\|_{\mathbb{H}}^{2}\right) \mathrm{d} s \\
& +\frac{1}{2} \int_{t}^{T} \mathbb{E}\|Z(s)-\tilde{Z}(s)\|_{L_{2}(\mathbb{U}, \mathbb{H})}^{2} \mathrm{~d} s .
\end{aligned}
$$

Hence, by Jensen's inequality

$$
\begin{aligned}
& \mathbb{E}\|Y(t)\|_{\mathbb{H}}^{2}+\frac{1}{2} \int_{t}^{T} \mathbb{E}\|Z(s)-\tilde{Z}(s)\|_{L_{2}(\mathbb{U}, \mathbb{H})}^{2} \mathrm{~d} s \\
\leqslant & c_{0} \int_{t}^{T} \mathbb{E}\|Y(s)\|_{\mathbb{H}}^{2} \mathrm{~d} s+\frac{1}{2} \int_{t}^{T} \rho\left(\mathbb{E}\|Y(s)\|_{\mathbb{H}}^{2}\right) \mathrm{d} s .
\end{aligned}
$$

The uniqueness follows from Lemma 2.2 .

Let us first prove the existence of solutions to Eq.(28) in the finite dimensional case. The following result is proved in [1]. Here we give it a simple proof by using Yosida's approximation. Compared with [1], our conditions are weaker than their conditions:

$$
\begin{array}{r}
X_{T} \in L^{2(q-1)}\left(\Omega, \mathcal{F}_{T}, P ; \mathbb{R}^{d}\right) \\
\mathbb{E}\left(\int_{0}^{T}|\eta(s)|^{2} \mathrm{~d} s\right)^{q-1}<+\infty .
\end{array}
$$

Lemma 4.3. Assume that $\mathbb{X}=\mathbb{H}=\mathbb{X}^{*}=\mathbb{U}=\mathbb{R}^{d}$ and $C=0$, and $(A, 0)$ satisfies $\mathscr{H}\left(0, \lambda_{1}, 0, \lambda_{3}, \xi, \eta, 0, q, 0\right)$, where $\lambda_{1}, \lambda_{3}$ are positive constants, $q \geqslant 2,0<\xi \in L^{1}(\mathfrak{A})$ and

$$
\mathbb{E}\left(\int_{0}^{T}|\eta(s)|^{2} \mathrm{~d} s\right)^{q / 2}<+\infty
$$

Then for any $X_{T} \in L^{q}\left(\Omega, \mathcal{F}_{T}, P ; \mathbb{R}^{d}\right)$, there exists a unique solution to Eq.(28) in the sense of Definition 4.1. Moreover,

$$
\mathbb{E}\left(\sup _{t \in[0, T]}\|X(t)\|_{\mathbb{R}^{d}}^{q}\right)+\mathbb{E}\left(\int_{0}^{T}\|Z(s)\|_{L_{2}\left(\mathbb{R}^{d}, \mathbb{R}^{d}\right)}^{2} \mathrm{~d} s\right)^{q / 2}
$$




$$
\leqslant c_{0} \cdot \mathbb{E}\left\|X_{T}\right\|_{\mathbb{R}^{d}}^{q}+c_{0} \cdot \mathbb{E}\left(\int_{0}^{T}|\eta(s)|^{2} \mathrm{~d} s\right)^{q / 2},
$$

where $c_{0}$ only depends on $q, T$ and $\lambda_{1}$.

Proof. For every $(t, \omega) \in[0, T] \times \Omega$, note that $x \mapsto A(t, \omega, x)$ is a continuous monotone function on $\mathbb{R}^{d}$. Let $A_{\varepsilon}(t, \omega, \cdot), \varepsilon>0$ be the Yosida approximation of $A(t, \omega, \cdot)$, i.e.:

$$
\begin{aligned}
A_{\varepsilon}(t, \omega, x) & :=\varepsilon^{-1}\left(J_{\varepsilon}(t, \omega, x)-x\right)=A\left(t, \omega, J_{\varepsilon}(t, \omega, x)\right), \\
J_{\varepsilon}(t, \omega, x) & :=(I-\varepsilon A(t, \omega, \cdot))^{-1}(x),
\end{aligned}
$$

then $x \mapsto J_{\varepsilon}(t, \omega, x)$ is a homeomorphism on $\mathbb{R}^{d}$ for each $(t, \omega)$ and for any $x, y \in \mathbb{R}^{d}$ (cf. [2] [5])

(I) $\left\langle x-y, A_{\varepsilon}(t, \omega, x)-A_{\varepsilon}(t, \omega, y)\right\rangle_{\mathbb{R}^{d}} \leqslant 0$,

(II) $\left\|A_{\varepsilon}(t, \omega, x)-A_{\varepsilon}(t, \omega, y)\right\|_{\mathbb{R}^{d}} \leqslant \varepsilon^{-1}\|x-y\|_{\mathbb{R}^{d}}$,

(III) $\left\|A_{\varepsilon}(t, \omega, x)\right\|_{\mathbb{R}^{d}} \leqslant\|A(t, \omega, x)\|_{\mathbb{R}^{d}}$,

(IV) $\lim _{\varepsilon \downarrow 0}\left\|A_{\varepsilon}(t, \omega, x)-A(t, \omega, x)\right\|_{\mathbb{R}^{d}}=0$.

By Lemma 2.6, $J_{\varepsilon}$ and $A_{\varepsilon}$ are progressively measurable. From (I), (III) and (H4), we have for any $x \in \mathbb{R}^{d}$

$$
\left\langle x, A_{\varepsilon}(t, \omega, x)\right\rangle_{\mathbb{R}^{d}} \leqslant\|x\|_{\mathbb{R}^{d}} \cdot\|A(t, \omega, 0)\|_{\mathbb{R}^{d}} \leqslant \eta(t, \omega) \cdot \lambda_{1}^{\frac{1}{q}} \cdot\|x\|_{\mathbb{R}^{d}} .
$$

Let $\left(X_{\varepsilon}, Z_{\varepsilon}\right)$ be the unique $\mathcal{F}_{t}$-adapted solution of the following backward stochastic differential equation(cf. [21])

$$
X_{\varepsilon}(t)=X_{T}+\int_{t}^{T} A_{\varepsilon}\left(s, X_{\varepsilon}(s)\right) \mathrm{d} s-\int_{t}^{T} Z_{\varepsilon}(s) \mathrm{d} W(s) .
$$

By Itô's formula, we have

$$
\begin{aligned}
& \left\|X_{\varepsilon}(t)\right\|_{\mathbb{R}^{d}}^{2}+\int_{t}^{T}\left\|Z_{\varepsilon}(s)\right\|_{L_{2}\left(\mathbb{R}^{d}, \mathbb{R}^{d}\right)}^{2} \mathrm{~d} s \\
= & \left\|X_{T}\right\|_{\mathbb{R}^{d}}^{2}+2 \int_{t}^{T}\left\langle X_{\varepsilon}(s), A_{\varepsilon}\left(s, X_{\varepsilon}(s)\right)\right\rangle_{\mathbb{R}^{d}} \mathrm{~d} s \\
& -2 \int_{t}^{T}\left\langle X_{\varepsilon}(s), Z_{\varepsilon}(s) \mathrm{d} W(s)\right\rangle_{\mathbb{R}^{d}}
\end{aligned}
$$

and further

$$
\begin{aligned}
& e^{t}\left\|X_{\varepsilon}(t)\right\|_{\mathbb{R}^{d}}^{2}+\int_{t}^{T} e^{s}\left(\left\|X_{\varepsilon}(s)\right\|_{\mathbb{R}^{d}}^{2}+\left\|Z_{\varepsilon}(s)\right\|_{L_{2}\left(\mathbb{R}^{d}, \mathbb{R}^{d}\right)}^{2}\right) \mathrm{d} s \\
= & e^{T}\left\|X_{T}\right\|_{\mathbb{R}^{d}}^{2}+2 \int_{t}^{T} e^{s}\left\langle X_{\varepsilon}(s), A_{\varepsilon}\left(s, X_{\varepsilon}(s)\right)\right\rangle_{\mathbb{R}^{d}} \mathrm{~d} s \\
& -2 \int_{t}^{T} e^{s}\left\langle X_{\varepsilon}(s), Z_{\varepsilon}(s) \mathrm{d} W(s)\right\rangle_{\mathbb{R}^{d}} \\
\leqslant & e^{T}\left\|X_{T}\right\|_{\mathbb{R}^{d}}^{2}+\int_{t}^{T} e^{s}\left\|X_{\varepsilon}(s)\right\|_{\mathbb{R}^{d}}^{2} \mathrm{~d} s+c_{0} \int_{t}^{T} e^{s}|\eta(s)|^{2} \mathrm{~d} s \\
& -2 \int_{t}^{T} e^{s}\left\langle X_{\varepsilon}(s), Z_{\varepsilon}(s) \mathrm{d} W(s)\right\rangle_{\mathbb{R}^{d}},
\end{aligned}
$$

where the second step is due to (30) and Young's inequality (5). 
Taking conditional expectations for both sides of (33) with respect to $\mathcal{F}_{t}$, we find

$$
\begin{aligned}
e^{t}\left\|X_{\varepsilon}(t)\right\|_{\mathbb{R}^{d}}^{2} & \leqslant e^{T} \mathbb{E}^{\mathcal{F}_{t}}\left\|X_{T}\right\|_{\mathbb{R}^{d}}^{2}+c_{0} \cdot \mathbb{E}^{\mathcal{F}_{t}} \int_{t}^{T} e^{s}|\eta(s)|^{2} \mathrm{~d} s \\
& \leqslant e^{T} \mathbb{E}^{\mathcal{F}_{t}}\left\|X_{T}\right\|_{\mathbb{R}^{d}}^{2}+c_{0} \cdot e^{T} \cdot \mathbb{E}^{\mathcal{F}_{t}} \int_{0}^{T}|\eta(s)|^{2} \mathrm{~d} s .
\end{aligned}
$$

Hence, by Doob's maximal inequality(cf. [25]), we have for $q>2$

$$
\mathbb{E}\left(\sup _{t \in[0, T]}\left\|X_{\varepsilon}(t)\right\|_{\mathbb{R}^{d}}^{q}\right) \leqslant c_{0} \cdot \mathbb{E}\left\|X_{T}\right\|_{\mathbb{R}^{d}}^{q}+c_{0} \cdot \mathbb{E}\left(\int_{0}^{T}|\eta(s)|^{2} \mathrm{~d} s\right)^{q / 2} .
$$

Hereafter $c_{0}$ only depends on $q, T$ and $\lambda_{1}$.

Noting that by BDG's inequality and Young's inequality (5)

$$
\begin{aligned}
& \mathbb{E}\left|\int_{0}^{T} e^{s}\left\langle X_{\varepsilon}(s), Z_{\varepsilon}(s) \mathrm{d} W(s)\right\rangle_{\mathbb{R}^{d}}\right|^{q / 2} \\
\leqslant & c_{0} \mathbb{E}\left(\int_{0}^{T} e^{2 s}\left\|X_{\varepsilon}(s)\right\|_{\mathbb{R}^{d}}^{2} \cdot\left\|Z_{\varepsilon}(s)\right\|_{L_{2}\left(\mathbb{R}^{d}, \mathbb{R}^{d}\right)}^{2} \mathrm{~d} s\right)^{q / 4} \\
\leqslant & c_{0} \mathbb{E}\left(\sup _{t \in[0, T]}\left\|X_{\varepsilon}(t)\right\|_{\mathbb{R}^{d}}^{q}\right)+\frac{1}{2} \mathbb{E}\left(\int_{0}^{T}\left\|Z_{\varepsilon}(s)\right\|_{L_{2}\left(\mathbb{R}^{d}, \mathbb{R}^{d}\right)}^{2} \mathrm{~d} s\right)^{q / 2},
\end{aligned}
$$

we also have from (33)

$$
\mathbb{E}\left(\int_{0}^{T}\left\|Z_{\varepsilon}(s)\right\|_{L_{2}\left(\mathbb{R}^{d}, \mathbb{R}^{d}\right)}^{2} \mathrm{~d} s\right)^{q / 2} \leqslant c_{0} \cdot \mathbb{E}\left\|X_{T}\right\|_{\mathbb{R}^{d}}^{q}+c_{0} \cdot \mathbb{E}\left(\int_{0}^{T}|\eta(s)|^{2} \mathrm{~d} s\right)^{q / 2} .
$$

For $q=2$, from (33) and the above proof, it is easy to see that

$$
\begin{aligned}
& \mathbb{E}\left(\sup _{t \in[0, T]}\left\|X_{\varepsilon}(t)\right\|_{\mathbb{R}^{d}}^{2}\right)+\int_{0}^{T} \mathbb{E}\left\|Z_{\varepsilon}(s)\right\|_{L_{2}\left(\mathbb{R}^{d}, \mathbb{R}^{d}\right)}^{2} \mathrm{~d} s \\
\leqslant & c_{0} \cdot \mathbb{E}\left\|X_{T}\right\|_{\mathbb{R}^{d}}^{2}+c_{0} \cdot \mathbb{E} \int_{0}^{T}|\eta(s)|^{2} \mathrm{~d} s .
\end{aligned}
$$

Moreover, by (III), (H4) and (34)

$$
\begin{aligned}
\int_{0}^{T}\left\|A_{\varepsilon}\left(s, X_{\varepsilon}(s)\right)\right\|_{\mathbb{R}^{d}}^{\frac{q}{q-1}} \mathrm{~d} s & \leqslant \int_{0}^{T}\left\|A\left(s, X_{\varepsilon}(s)\right)\right\|_{\mathbb{R}^{d}}^{\frac{q}{q-1}} \mathrm{~d} s \\
& \leqslant c_{0} \int_{0}^{T}\left\|X_{\varepsilon}(s)\right\|_{\mathbb{R}^{d}}^{q} \mathrm{~d} s+c_{0} \int_{0}^{T} \mathbb{E}\left(\eta^{\frac{q}{q-1}}(s)\right) \mathrm{d} s \leqslant c_{0}^{\prime} .
\end{aligned}
$$

Therefore, there exists a subsequence $\varepsilon_{n} \downarrow 0$ and $\left(X, Y, Z, X_{0}\right)$ such that

$$
\begin{aligned}
X_{\varepsilon_{n}} & \rightarrow X \text { weakly in } \mathbb{K}_{2,1}, \\
A_{\varepsilon_{n}}\left(\cdot, X_{\varepsilon_{n}}(\cdot)\right) & \rightarrow Y \text { weakly in } \mathbb{K}_{1,1}, \\
Z_{\varepsilon_{n}} & \rightarrow Z \text { weakly in } \mathbb{K}_{3}, \\
X_{\varepsilon_{n}}(0) & \rightarrow X_{0} \text { weakly in } L^{2}\left(\Omega, \mathcal{F}_{0}, P ; \mathbb{R}^{d}\right)
\end{aligned}
$$

as $n \rightarrow \infty$. By (34) and (35), we get (29).

Set

$$
\tilde{X}(t):=X_{T}+\int_{t}^{T} Y(s) \mathrm{d} s-\int_{t}^{T} Z(s) \mathrm{d} W(s) .
$$


By taking weak limits for (31), we deduce that $\tilde{X}(0)=X_{0}$ a.s. and

$$
X(t, \omega)=\tilde{X}(t, \omega) \text { for almost all }(t, \omega) \in[0, T] \times \Omega .
$$

It remains to show that $Y(s)=A(s, X(s))$. For any $\Phi \in \mathbb{K}_{2,1}$, by (III) (IV) and the dominated convergence theorem we have

$$
\begin{aligned}
& \lim _{n \rightarrow \infty} \mathbb{E}\left(\int_{0}^{T}\left\langle X_{\varepsilon_{n}}(s)-\Phi(s), A_{\varepsilon_{n}}(s, \Phi(s))-A(s, \Phi(s))\right\rangle_{\mathbb{R}^{d}} \mathrm{~d} s\right) \\
\leqslant & \lim _{n \rightarrow \infty}\left(\left\|A_{\varepsilon_{n}}(\cdot, \Phi(\cdot))-A(\cdot, \Phi(\cdot))\right\|_{\mathbb{K}_{1,1}}^{\frac{q}{q-1}} \cdot\left\|X_{\varepsilon_{n}}-\Phi\right\|_{L^{q}(\mathfrak{A})}\right)=0 .
\end{aligned}
$$

On the other hand, we have by (32)

$$
\begin{aligned}
& 2 \liminf _{n \rightarrow \infty} \mathbb{E}\left(\int_{0}^{T}\left\langle X_{\varepsilon_{n}}(s), A_{\varepsilon_{n}}\left(s, X_{\varepsilon_{n}}(s)\right)\right\rangle_{\mathbb{R}^{d}} \mathrm{~d} s\right) \\
\geqslant & \mathbb{E}\left\|X_{0}\right\|_{\mathbb{R}^{d}}^{2}-\mathbb{E}\left\|X_{T}\right\|_{\mathbb{R}^{d}}^{2}+\int_{0}^{T} \mathbb{E}\|Z(s)\|_{L_{2}\left(\mathbb{R}^{d}, \mathbb{R}^{d}\right)}^{2} \mathrm{~d} s \\
= & \left.2 \mathbb{E}\left(\int_{0}^{T}\langle X(s), Y(s))\right\rangle_{\mathbb{R}^{d}} \mathrm{~d} s\right) .
\end{aligned}
$$

Combining (36) and (37), we have by (I)

$$
\begin{aligned}
& \left.\mathbb{E}\left(\int_{0}^{T}\langle X(s)-\Phi(s), Y(s)-A(s, \Phi(s)))\right\rangle_{\mathbb{R}^{d}} \mathrm{~d} s\right) \\
\leqslant & \liminf _{n \rightarrow \infty} \mathbb{E}\left(\int_{0}^{T}\left\langle X_{\varepsilon_{n}}(s)-\Phi(s), A_{\varepsilon_{n}}\left(s, X_{\varepsilon_{n}}(s)\right)-A_{\varepsilon_{n}}(s, \Phi(s))\right\rangle_{\mathbb{R}^{d}} \mathrm{~d} s\right) \leqslant 0,
\end{aligned}
$$

which implies that $Y=A(\cdot, X)$ by Lemma 2.5. The proof is complete.

Remark 4.4. When $q>2$, it suffices to require that

$$
\mathbb{E}\left(\int_{0}^{T}|\eta(s)| \mathrm{d} s\right)^{q}<+\infty .
$$

In fact, taking conditional expectations for both sides of (32) with respect to $\mathcal{F}_{t}$, and by (30) and Young's inequality (5) we find for any $\delta>0$

$$
\begin{aligned}
\left\|X_{\varepsilon}(t)\right\|_{\mathbb{R}^{d}}^{2} \leqslant & \mathbb{E}^{\mathcal{F}_{t}}\left\|X_{T}\right\|_{\mathbb{R}^{d}}^{2}+q \mathbb{E}^{\mathcal{F}_{t}}\left(\int_{t}^{T}\left\|X_{\varepsilon}(s)\right\|_{\mathbb{R}^{d}} \cdot \eta(s) \cdot \lambda_{1}^{\frac{1}{q}} \mathrm{~d} s\right) \\
\leqslant & \mathbb{E}^{\mathcal{F}_{t}}\left\|X_{T}\right\|_{\mathbb{R}^{d}}^{2}+\delta \cdot \mathbb{E}^{\mathcal{F}_{t}}\left(\sup _{s \in[0, T]}\left\|X_{\varepsilon}(s)\right\|_{\mathbb{R}^{d}}^{2}\right) \\
& +c_{\delta} \cdot \mathbb{E}^{\mathcal{F}_{t}}\left(\int_{0}^{T}|\eta(s)| \mathrm{d} s\right)^{2} .
\end{aligned}
$$

Hence, by Doob's maximal inequality we have for $q>2$

$$
\begin{aligned}
\mathbb{E}\left(\sup _{t \in[0, T]}\left\|X_{\varepsilon}(t)\right\|_{\mathbb{R}^{d}}^{q}\right) \leqslant & c_{0} \cdot \mathbb{E}\left\|X_{T}\right\|_{\mathbb{R}^{d}}^{q}+\delta \cdot c_{q} \cdot \mathbb{E}\left(\sup _{s \in[0, T]}\left\|X_{\varepsilon}(s)\right\|_{\mathbb{R}^{d}}^{q}\right) \\
& +c_{\delta} \cdot \mathbb{E}\left(\int_{01}^{T}|\eta(s)| \mathrm{d} s\right)^{q} .
\end{aligned}
$$


Letting $\delta$ be sufficiently small, we get

$$
\mathbb{E}\left(\sup _{t \in[0, T]}\left\|X_{\varepsilon}(t)\right\|_{\mathbb{R}^{d}}^{q}\right) \leqslant c_{0} \cdot \mathbb{E}\left\|X_{T}\right\|_{\mathbb{R}^{d}}^{q}+c_{0} \cdot \mathbb{E}\left(\int_{0}^{T}|\eta(s)| \mathrm{d} s\right)^{q},
$$

where $c_{0}$ only depends on $q, \lambda_{1}$ and $T$.

We now prove the following infinite dimensional version.

Lemma 4.5. Assume that $C(\cdot, x, z)=C(\cdot) \in L^{2}\left(\mathfrak{A}_{a} ; \mathbb{H}\right)$ is independent of $x$ and $z$, and (HB1) holds. Then there exists a unique solution to Eq.(28) in the sense of Definition 4.1.

Proof. We use Galerkin's approximation to prove the existence as in the proof of Theorem 3.6. For $n \in \mathbb{N}$, let $\left(X_{n}, Z_{n}\right)$ solve the following finite dimensional backward stochastic differential equation (Lemma 4.3)

$$
X_{n}(t)=X_{T}^{n}+\int_{t}^{T} \Pi_{n} A\left(s, X_{n}(s)\right) \mathrm{d} s+\int_{t}^{T} C_{n}(s) \mathrm{d} s-\int_{t}^{T} Z_{n}(s) \tilde{\Pi}_{n} \mathrm{~d} W(s),
$$

where $\Pi_{n}$ and $\tilde{\Pi}_{n}$ are same as in Theorem [3.6, and

$$
\begin{aligned}
X_{T}^{n} & :=\Pi_{n} X_{T} \cdot 1_{\left\{\left\|\Pi_{n} X_{T}\right\|_{\mathbb{H}} \leqslant n\right\}} \\
C_{n}(s) & :=\Pi_{n} C(s) \cdot 1_{\left\{\left\|\Pi_{n} C(s)\right\|_{H} \leqslant n\right\}} .
\end{aligned}
$$

It is easy to see that for each $n$ and $s$

$$
\left\|X_{T}^{n}\right\|_{\mathbb{H}} \leqslant\left\|X_{T}\right\|_{\mathbb{H}}, \quad\left\|C_{n}(s)\right\|_{\mathbb{H}} \leqslant\|C(s)\|_{\mathbb{H}}
$$

and

$$
\begin{aligned}
\lim _{n \rightarrow \infty} \mathbb{E}\left\|X_{T}^{n}-X_{T}\right\|_{\mathbb{H}}^{2} & =0, \\
\lim _{n \rightarrow \infty} \int_{0}^{T} \mathbb{E}\left\|C_{n}(s)-C(s)\right\|_{\mathbb{H}}^{2} \mathrm{~d} s & =0 .
\end{aligned}
$$

By Itô's formula and (H3), we have

$$
\begin{aligned}
& \mathbb{E}\left\|X_{n}(t)\right\|_{\mathbb{H}}^{2}+\int_{t}^{T} \mathbb{E}\left\|Z_{n}(s)\right\|_{L_{2}(\mathbb{U}, \mathbb{H})}^{2} \mathrm{~d} s \\
= & \mathbb{E}\left\|X_{T}^{n}\right\|_{\mathbb{H}}^{2}+\int_{t}^{T} \mathbb{E}\left[X_{n}(s), A\left(s, X_{n}(s)\right)\right]_{\mathbb{X}} \mathrm{d} s+2 \int_{t}^{T} \mathbb{E}\left\langle X_{n}(s), C_{n}(s)\right\rangle_{\mathbb{H}} \mathrm{d} s \\
\leqslant & \mathbb{E}\left\|X_{T}\right\|_{\mathbb{H}}^{2}+\int_{0}^{T} \mathbb{E}\left(2 \xi(s)+\|C(s)\|_{\mathbb{H}}^{2}\right) \mathrm{d} s \\
& +\int_{t}^{T} \mathbb{E}\left(-\sum_{i=1,2} \lambda_{i} \cdot\left\|X_{n}(s)\right\|_{\mathbb{X}_{i}}^{q_{i}}+\left(\lambda_{3}+1\right) \cdot\left\|X_{n}(s)\right\|_{\mathbb{H}}^{2}\right) \mathrm{d} s .
\end{aligned}
$$

By Gronwall's inequality we have

$$
\mathbb{E}\left\|X_{n}(t)\right\|_{\mathbb{H}}^{2} \leqslant c_{0}\left(\mathbb{E}\left\|X_{T}\right\|_{\mathbb{H}}^{2}+\int_{0}^{T} \mathbb{E}\left(\xi(s)+\|C(s)\|_{\mathbb{H}}^{2}\right) \mathrm{d} s\right) .
$$

Hence, from (41) and (H4) we get

$$
\mathbb{E}\left\|X_{n}(0)\right\|_{\mathbb{H}}^{2}+\left\|X_{n}\right\|_{\mathbb{K}_{4}}^{2}+\left\|Z_{n}\right\|_{\mathbb{K}_{3}}^{2}+\sum_{i=1,2}\left(\left\|X_{n}\right\|_{\mathbb{K}_{2, i}}^{q_{i}}+\left\|A_{i}\left(\cdot, X_{n}\right)\right\|_{\mathbb{K}_{1, i}}^{\frac{q_{i}}{q_{i}-1}}\right) \leqslant c_{0} .
$$

Hereafter, the constant $c_{0}$ is independent of $n$. 
By the reflexivities of Banach spaces $\mathbb{K}$, one may find a subsequence $n_{k}$ (denoted by $k$ for simplicity) and $\tilde{X} \in \mathbb{K}_{2,1} \cap \mathbb{K}_{2,2} \cap \mathbb{K}_{4}, Y_{i} \in \mathbb{K}_{1, i}, i=1,2$ and $Z \in \mathbb{K}_{3}$ such that

$$
\begin{aligned}
X_{k} & \rightarrow \tilde{X} \text { weakly in } \mathbb{K}_{2,1}, \mathbb{K}_{2,2} \text { and } \mathbb{K}_{4}, \\
A_{i}\left(\cdot, X_{k}\right)=: Y_{k, i} & \rightarrow Y_{i} \text { weakly in } \mathbb{K}_{1, i}, \quad i=1,2, \\
Z_{k} & \rightarrow Z \text { weakly in } \mathbb{K}_{3}, \\
X_{k}(0) & \rightarrow X_{0} \text { weakly in } L^{2}\left(\Omega, \mathcal{F}_{0}, P ; \mathbb{H}\right) .
\end{aligned}
$$

Define $Y=Y_{1}+Y_{2} \in \mathbb{X}^{*}$ and

$$
X(t):=X_{T}+\int_{t}^{T} Y(s) \mathrm{d} s+\int_{t}^{T} C(s) \mathrm{d} s-\int_{t}^{T} Z(s) \mathrm{d} W(s) .
$$

Then, similar to Step 3 of Theorem 3.6 one may prove that

$$
\begin{aligned}
\tilde{X}(t, \omega) & =X(t, \omega) \quad \text { for }(\mathrm{d} t \times \mathrm{d} P) \text {-almost all }(t, \omega), \\
X(0) & =X_{0} \quad \text { a.s.. }
\end{aligned}
$$

We now show that

$$
A(t, X(t, \omega))=Y(t, \omega) \quad \text { for }(\mathrm{d} t \times \mathrm{d} P) \text {-almost all }(t, \omega) .
$$

By (401) and (H2)(with $\lambda_{0}=0$ ), we have for any $\Phi \in \mathbb{K}_{2}$

$$
\begin{aligned}
& \mathbb{E}\left\|X_{k}(0)\right\|_{\mathbb{H}}^{2}+\int_{0}^{T} \mathbb{E}\left\|Z_{k}(s)\right\|_{L_{2}(\mathbb{U}, \mathbb{H})}^{2} \mathrm{~d} s \\
\leqslant & \mathbb{E}\left\|X_{T}^{k}\right\|_{\mathbb{H}}^{2}+2 \int_{0}^{T} \mathbb{E}\left[\Phi(s), A\left(s, X_{k}(s)\right)-A(s, \Phi(s))\right]_{\mathbb{X}} \mathrm{d} s \\
& +2 \int_{0}^{T} \mathbb{E}\left[X_{k}(s), A(s, \Phi(s))\right]_{\mathbb{X}} \mathrm{d} s+2 \int_{0}^{T} \mathbb{E}\left\langle X_{k}(s), C_{k}(s)\right\rangle_{\mathbb{H}} \mathrm{d} s .
\end{aligned}
$$

Taking limits for $k \rightarrow \infty$ and by (38) (39), we find

$$
\begin{aligned}
& \liminf _{k \rightarrow \infty} \mathbb{E}\left\|X_{k}(0)\right\|_{\mathbb{H}}^{2}+\liminf _{k \rightarrow \infty} \int_{0}^{T} \mathbb{E}\left\|Z_{k}(s)\right\|_{L_{2}(\mathbb{U}, \mathbb{H})}^{2} \mathrm{~d} s \\
\leqslant & \mathbb{E}\left\|X_{T}\right\|_{\mathbb{H}}^{2}+2 \int_{0}^{T} \mathbb{E}[\Phi(s), Y(s)-A(s, \Phi(s))]_{\mathbb{X}} \mathrm{d} s \\
& +2 \int_{0}^{T} \mathbb{E}[X(s), A(s, \Phi(s))]_{\mathbb{X}} \mathrm{d} s+2 \int_{0}^{T} \mathbb{E}\langle X(s), C(s)\rangle_{\mathbb{H}} \mathrm{d} s .
\end{aligned}
$$

On the other hand, noting that

$$
\begin{aligned}
& \mathbb{E}\left\|X_{0}\right\|_{\mathbb{H}}^{2}+\int_{0}^{T} \mathbb{E}\|Z(s)\|_{L_{2}(\mathbb{U}, \mathbb{H})}^{2} \mathrm{~d} s \\
= & \mathbb{E}\left\|X_{T}\right\|_{\mathbb{H}}^{2}+2 \int_{0}^{T} \mathbb{E}[X(s), Y(s)]_{\mathbb{X}} \mathrm{d} s+2 \int_{0}^{T} \mathbb{E}\langle X(s), C(s)\rangle_{\mathbb{H}} \mathrm{d} s
\end{aligned}
$$

and

$$
\begin{aligned}
\mathbb{E}\left\|X_{0}\right\|_{\mathbb{H}}^{2} & \leqslant \liminf _{k \rightarrow \infty} \mathbb{E}\left\|X_{k}(0)\right\|_{\mathbb{H}}^{2}, \\
\int_{0}^{T} \mathbb{E}\|Z(s)\|_{L_{2}(\mathbb{U}, \mathbb{H})}^{2} \mathrm{~d} s & \leqslant \liminf _{\substack{k \rightarrow \infty \\
23}} \int_{0}^{T} \mathbb{E}\left\|Z_{k}(s)\right\|_{L_{2}(\mathbb{U}, \mathbb{H})}^{2} \mathrm{~d} s
\end{aligned}
$$


we obtain

$$
\int_{0}^{T} \mathbb{E}[X(s)-\Phi(s), Y(s)-A(s, \Phi(s))]_{\mathbb{X}} \mathrm{d} s \leqslant 0 .
$$

Hence $Y=A(\cdot, X)$ by Lemma 2.5, The proof is complete.

Lemma 4.6. Assume that $C(t, x, z)=C(t, z)$ is independent of $x$, and (HB1), (HB2) and (HB3) hold. Then there exists a unique solution to Eq.(28) in the sense of Definition 4.1.

Proof. Let $Z_{0}(t) \equiv 0$. We consider the following Picard iteration: for $n \in \mathbb{N}$, let $\left(X_{n}, Z_{n}\right)$ solve the following equation(Lemma 4.5):

$$
X_{n}(t)=X_{T}+\int_{t}^{T} A\left(s, X_{n}(s)\right) \mathrm{d} s+\int_{t}^{T} C\left(s, Z_{n-1}(s)\right) \mathrm{d} s-\int_{t}^{T} Z_{n}(s) \mathrm{d} W(s) .
$$

Set $Y_{n}(t):=X_{n+1}(t)-X_{n}(t)$. By Itô's formula, (H2)(with $\left.\lambda_{0}=0\right),(\mathbf{H B 2})$ and Young's inequality, we have

$$
\begin{aligned}
& \mathbb{E}\left\|Y_{n}(t)\right\|_{\mathbb{H}}^{2}+\int_{t}^{T} \mathbb{E}\left\|Z_{n+1}(s)-Z_{n}(s)\right\|_{L_{2}(\mathbb{U}, \mathbb{H})}^{2} \mathrm{~d} s \\
= & \int_{t}^{T} \mathbb{E}\left[Y_{n}(s), A\left(s, X_{n+1}(s)\right)-A\left(s, X_{n}(s)\right)\right]_{\mathbb{X}} \mathrm{d} s \\
& +\int_{t}^{T} \mathbb{E}\left\langle Y_{n}(s), C\left(s, Z_{n}(s)\right)-C\left(s, Z_{n-1}(s)\right)\right\rangle_{\mathbb{H}} \mathrm{d} s \\
\leqslant & c_{0} \int_{t}^{T} \mathbb{E}\left\|Y_{n}(s)\right\|_{\mathbb{H}}^{2} \mathrm{~d} s+\frac{1}{2} \int_{t}^{T}\left\|Z_{n}(s)-Z_{n-1}(s)\right\|_{L_{2}(\mathbb{U}, \mathbb{H})}^{2} \mathrm{~d} s .
\end{aligned}
$$

Hence, for $\alpha:=c_{0}$

$$
\begin{aligned}
& -\frac{\mathrm{d}}{\mathrm{d} t}\left(e^{\alpha t} \int_{t}^{T} \mathbb{E}\left\|Y_{n}(s)\right\|_{\mathbb{H}}^{2} \mathrm{~d} s\right)+e^{\alpha t} \int_{t}^{T} \mathbb{E}\left\|Z_{n+1}(s)-Z_{n}(s)\right\|_{L_{2}(\mathbb{U}, \mathbb{H})}^{2} \mathrm{~d} s \\
\leqslant & \frac{e^{\alpha t}}{2} \int_{t}^{T}\left\|Z_{n}(s)-Z_{n-1}(s)\right\|_{L_{2}(\mathbb{U}, \mathbb{H})}^{2} \mathrm{~d} s=: \frac{g_{n}(t)}{2} .
\end{aligned}
$$

Integrating both sides from 0 to $T$ yields that

$$
\int_{0}^{T} \mathbb{E}\left\|Y_{n}(s)\right\|_{\mathbb{H}}^{2} \mathrm{~d} s+\int_{0}^{T} g_{n+1}(t) \mathrm{d} t \leqslant \frac{1}{2} \int_{0}^{T} g_{n}(t) \mathrm{d} t \leqslant \frac{1}{2^{n}} \int_{0}^{T} g_{1}(t) \mathrm{d} t=: \frac{c_{0}}{2^{n}} .
$$

It then follows from (43) that

$$
\int_{0}^{T} \mathbb{E}\left\|Z_{n+1}(s)-Z_{n}(s)\right\|_{L_{2}(\mathbb{U}, \mathbb{H})}^{2} \mathrm{~d} s \leqslant \frac{c_{0}}{2^{n}}+\frac{1}{2} \int_{0}^{T} \mathbb{E}\left\|Z_{n}(s)-Z_{n-1}(s)\right\|_{L_{2}(\mathbb{U}, \mathbb{H})}^{2} \mathrm{~d} s .
$$

Iterating this inequality gives

$$
\int_{0}^{T} \mathbb{E}\left\|Z_{n+1}(s)-Z_{n}(s)\right\|_{L_{2}(\mathbb{U}, \mathbb{H})}^{2} \mathrm{~d} s \leqslant \frac{n c_{0}}{2^{n}} .
$$

Therefore, there exist an $X \in \mathbb{K}_{4}$ and a $Z \in \mathbb{K}_{3}$ such that

$$
\lim _{n \rightarrow \infty}\left\|X_{n}-X\right\|_{\mathbb{K}_{4}}=0 \text { and } \lim _{n \rightarrow \infty}\left\|Z_{n}-Z\right\|_{\mathbb{K}_{3}}=0 .
$$

From (43) and the above estimates, we also have

$$
\sup _{n \in \mathbb{N}} \sup _{t \in[0, T]} \mathbb{E}\left\|X_{n}(t)\right\|_{\mathbb{H}}^{2}<+\infty \text {. }
$$


We now show that there exists a version $(\tilde{X}, \tilde{Z})$ of $(X, Z)$ such that $(\tilde{X}, \tilde{Z})$ is a solution to Eq.(28) in the sense of Definition 4.1. In fact, let $(\tilde{X}, \tilde{Z})$ solve the following equation(Lemma 4.5):

$$
\tilde{X}(t)=X_{T}+\int_{t}^{T} A(s, \tilde{X}(s)) \mathrm{d} s+\int_{t}^{T} C(s, Z(s)) \mathrm{d} s-\int_{t}^{T} \tilde{Z}(s) \mathrm{d} W(s) .
$$

It is similar to estimate (43) that

$$
\begin{aligned}
& \mathbb{E}\left\|X_{n}(t)-\tilde{X}(t)\right\|_{\mathbb{H}}^{2}+\int_{t}^{T} \mathbb{E}\left\|Z_{n}(s)-\tilde{Z}(s)\right\|_{L_{2}(\mathbb{U}, \mathbb{H})}^{2} \mathrm{~d} s \\
\leqslant & c_{0} \int_{t}^{T} \mathbb{E}\left\|X_{n}(s)-\tilde{X}(s)\right\|_{\mathbb{H}}^{2} \mathrm{~d} s+\frac{1}{2} \int_{t}^{T}\left\|Z_{n-1}(s)-Z(s)\right\|_{L_{2}(\mathbb{U}, \mathbb{H})}^{2} \mathrm{~d} s .
\end{aligned}
$$

Letting $g(t):=\limsup _{n \rightarrow \infty} \mathbb{E}\left\|X_{n}(t)-\tilde{X}(t)\right\|_{\mathbb{H}}^{2}$, by (44) and Fatou's lemma, we have

$$
g(t) \leqslant c_{0} \int_{t}^{T} g(s) \mathrm{d} s
$$

which yields that $g(t)=0$ by Gronwall's inequality. The proof is complete.

We now prove our main result in this section.

Theorem 4.7. Assume that (HB1), (HB2) and (HB3) hold. Then there exists a unique solution to Eq.(28) in the sense of Definition 4.1.

Proof. Let $X_{0}(t) \equiv 0$. We consider the following Picard iteration: for $n \in \mathbb{N}$, let $\left(X_{n}, Z_{n}\right)$ solve the following equation(Lemma 4.6)

$$
X_{n}(t)=X_{T}+\int_{t}^{T} A\left(s, X_{n}(s)\right) \mathrm{d} s+\int_{t}^{T} C\left(s, X_{n-1}(s), Z_{n}(s)\right) \mathrm{d} s-\int_{t}^{T} Z_{n}(s) \mathrm{d} W(s) .
$$

First of all, by Itô's formula, (H2)(with $\left.\lambda_{0}=0\right),($ HB3) and Young's inequality, we have

$$
\begin{aligned}
& \mathbb{E}\left\|X_{n}(t)\right\|_{\mathbb{H}}^{2}+\int_{t}^{T} \mathbb{E}\left\|Z_{n}(s)\right\|_{L_{2}(\mathbb{U}, \mathbb{H})}^{2} \mathrm{~d} s \\
= & \mathbb{E}\left\|X_{T}\right\|_{\mathbb{H}}^{2}+2 \int_{t}^{T} \mathbb{E}\left[X_{n}(s), A\left(s, X_{n}(s)\right)\right]_{\mathbb{X}} \mathrm{d} s \\
& \left.+2 \int_{t}^{T} \mathbb{E}\left\langle X_{n}(s), C\left(s, X_{n-1}(s), Z_{n}(s)\right)\right)\right\rangle_{\mathbb{H}} \mathrm{d} s \\
\leqslant & \mathbb{E}\left\|X_{T}\right\|_{\mathbb{H}}^{2}+2 \int_{t}^{T} \mathbb{E}\left(\lambda_{3}\left\|X_{n}(s)\right\|_{\mathbb{H}}^{2}+\xi(s)\right) \mathrm{d} s \\
& +2 \int_{t}^{T} \mathbb{E}\left(\left\|X_{n}(s)\right\|_{\mathbb{H}}\left(\zeta(s)+c_{2}\left(\left\|X_{n-1}(s)\right\|_{\mathbb{H}}+\left\|Z_{n}(s)\right\|_{L_{2}(\mathbb{U}, \mathbb{H})}\right)\right)\right) \mathrm{d} s \\
\leqslant & \mathbb{E}\left\|X_{T}\right\|_{\mathbb{H}}^{2}+c_{0} \int_{0}^{T} \mathbb{E}\left(\xi(s)+\zeta^{2}(s)\right) \mathrm{d} s+c_{0} \int_{t}^{T} \mathbb{E}\left\|X_{n}(s)\right\|_{\mathbb{H}}^{2} \mathrm{~d} s \\
& +\frac{1}{4} \int_{t}^{T} \mathbb{E}\left(\left\|X_{n-1}(s)\right\|_{\mathbb{H}}^{2}+\left\|Z_{n}(s)\right\|_{L_{2}(\mathbb{U}, \mathbb{H})}^{2}\right) \mathrm{d} s .
\end{aligned}
$$

So

$$
\mathbb{E}\left\|X_{n}(t)\right\|_{\mathbb{H}}^{2}+\int_{t}^{T} \mathbb{E}\left\|Z_{n}(s)\right\|_{L_{2}(\mathbb{U}, \mathbb{H})}^{2} \mathrm{~d} s
$$




$$
\leqslant c_{0}+c_{0} \int_{t}^{T}\left(\mathbb{E}\left\|X_{n}(s)\right\|_{\mathbb{H}}^{2}+\mathbb{E}\left\|X_{n-1}(s)\right\|_{\mathbb{H}}^{2}\right) \mathrm{d} s,
$$

where $c_{0}$ is independent of $n$.

Set

$$
g_{n}(t):=\max _{1 \leqslant k \leqslant n} \mathbb{E}\left\|X_{k}(t)\right\|_{\mathbb{H}}^{2}
$$

Then

$$
g_{n}(t) \leqslant c_{0}+c_{0} \int_{t}^{T} g_{n}(s) \mathrm{d} s
$$

which gives that by Gronwall's inequality

$$
\max _{k \in \mathbb{N}} \sup _{t \in[0, T]} \mathbb{E}\left\|X_{k}(t)\right\|_{\mathbb{H}}^{2} \leqslant \max _{n \in \mathbb{N}} \sup _{t \in[0, T]} g_{n}(t)<+\infty .
$$

Set $Y_{n, m}(t):=X_{n}(t)-X_{m}(t)$ and $G_{n, m}(t):=Z_{n}(s)-Z_{m}(s)$. By Itô's formula, $(\mathbf{H 2})$ (with $\left.\lambda_{0}=0\right)$ and (HB2), we have

$$
\begin{aligned}
& \mathbb{E}\left\|Y_{n, m}(t)\right\|_{\mathbb{H}}^{2}+\int_{t}^{T} \mathbb{E}\left\|G_{n, m}(s)\right\|_{L_{2}(\mathbb{U}, \mathbb{H})}^{2} \mathrm{~d} s \\
= & 2 \int_{t}^{T} \mathbb{E}\left[Y_{n, m}(s), A\left(s, X_{n}(s)\right)-A\left(s, X_{m}(s)\right)\right]_{\mathbb{X}} \mathrm{d} s \\
& +2 \int_{t}^{T} \mathbb{E}\left\langle Y_{n, m}(s), C\left(s, X_{n-1}(s), Z_{n}(s)\right)-C\left(s, X_{m-1}(s), Z_{m}(s)\right)\right\rangle_{\mathbb{H}} \mathrm{d} s \\
\leqslant & c_{0} \int_{t}^{T} \mathbb{E}\left(\left\|Y_{n, m}(s)\right\|_{\mathbb{H}}\left(\rho\left(\left\|Y_{n-1, m-1}(s)\right\|_{\mathbb{H}}^{2}\right)+\left\|G_{n, m}(s)\right\|_{L_{2}(\mathbb{U}, \mathbb{H})}^{2}\right)^{\frac{1}{2}}\right) \mathrm{d} s .
\end{aligned}
$$

Using the same method as in estimating (45), we have

$$
\begin{aligned}
& \mathbb{E}\left\|Y_{n, m}(t)\right\|_{\mathbb{H}}^{2}+\int_{t}^{T} \mathbb{E}\left\|G_{n, m}(s)\right\|_{L_{2}(\mathbb{U}, \mathbb{H})}^{2} \mathrm{~d} s \\
\leqslant & c_{0} \int_{t}^{T} \mathbb{E}\left\|Y_{n, m}(s)\right\|_{\mathbb{H}}^{2} \mathrm{~d} s+c_{0} \int_{t}^{T} \mathbb{E} \rho\left(\left\|Y_{n-1, m-1}(s)\right\|_{\mathbb{H}}^{2}\right) \mathrm{d} s .
\end{aligned}
$$

Set

$$
g(t):=\limsup _{n, m \rightarrow \infty} \mathbb{E}\left\|Y_{n, m}(t)\right\|_{\mathbb{H}}^{2} \cdot
$$

By (46), Fatou's lemma and Jensen's inequality, we have

$$
g(t) \leqslant c_{0} \int_{t}^{T}(g(s)+\rho(g(s))) \mathrm{d} s .
$$

So, by Lemma 2.2

$$
g(t)=0, \quad t \in[0, T]
$$

Hence

$$
\limsup _{n, m \rightarrow \infty} \int_{0}^{T}\left(\mathbb{E}\left\|Y_{n, m}(s)\right\|_{\mathbb{H}}^{2}+\mathbb{E}\left\|G_{n, m}(s)\right\|_{L_{2}(\mathbb{U}, \mathbb{H})}^{2}\right) \mathrm{d} s=0,
$$

and there exist an $X \in \mathbb{K}_{4}$ and a $Z \in \mathbb{K}_{3}$ such that

$$
\lim _{n \rightarrow \infty}\left\|X_{n}-X\right\|_{\mathbb{K}_{4}}=0 \text { and } \lim _{n \rightarrow \infty}\left\|Z_{n}-Z\right\|_{\mathbb{K}_{3}}=0 .
$$


Using the same method as in the proof of Lemma 4.6, we can show that $(X, Z)$ solves Eq.(28). The proof is thus complete.

\section{Stochastic Functional Integral Evolution Equations}

Fix $S>0$. For any $T \geqslant 0$, let $\mathbb{F}_{S}^{T}(\mathbb{H})$ denote the space of all continuous functions from $[-S, T]$ to $\mathbb{H}$, which is a separable Banach space under the supremum norm

$$
\|f\|_{\mathbb{F}_{S}^{T}}:=\sup _{s \in[-S, T]}\|f(s)\|_{\mathbb{H} \cdot}
$$

For $s \in[-S, 0]$, define $\mathcal{F}_{s}:=\mathcal{F}_{0}$. Suppose that $X:[-S, T] \times \Omega \mapsto \mathbb{H}$ is a continuous $\mathcal{F}_{t^{-}}$adapted process, we can associate it with another continuous $\mathbb{F}_{S}^{0}(\mathbb{H})$-valued and $\mathcal{F}_{t^{-}}$ adapted process as follows:

$$
[0, T] \times \Omega \ni(t, \omega) \mapsto X_{t}(\cdot, \omega):=X(t+\cdot, \omega) \in \mathbb{F}_{S}^{0}(\mathbb{H}) .
$$

In the following, we shall use the following notations:

$$
\|X .(0)\|_{\mathbb{F}_{0}^{t}}:=\sup _{s \in[0, t]}\left\|X_{s}(0)\right\|_{\mathbb{H}}=\sup _{s \in[0, t]}\|X(s)\|_{\mathbb{H}}=:\|X\|_{\mathbb{F}_{0}^{t}} .
$$

Consider the following stochastic functional integral evolution equation:

$$
\begin{aligned}
X_{t}(0)= & X_{0}(0)+\int_{0}^{t} A\left(s, X_{s}(0)\right) \mathrm{d} s+\int_{0}^{t} C_{1}\left(s, X_{s}\right) \mathrm{d} s+\int_{0}^{t} \int_{0}^{s} C_{2}\left(s, r, X_{r}\right) \mathrm{d} r \mathrm{~d} s \\
& +\int_{0}^{t} D_{1}\left(s, X_{s}\right) \mathrm{d} W_{s}+\int_{0}^{t} \int_{0}^{s} D_{2}\left(s, r, X_{r}\right) \mathrm{d} W_{r} \mathrm{~d} s,
\end{aligned}
$$

where $X_{0}$ is an $\mathcal{F}_{0}$-measurable $\mathbb{F}_{S}^{0}(\mathbb{H})$-valued random variable and $A=A_{1}+A_{2}$,

$$
\begin{aligned}
A_{i}:[0, T] \times \Omega \times \mathbb{X}_{i} & \mapsto \mathbb{X}_{i}^{*}, \quad i=1,2, \\
C_{1}:[0, T] \times \Omega \times \mathbb{F}_{S}^{0}(\mathbb{H}) & \mapsto \mathbb{H}, \\
C_{2}:[0, T] \times[0, T] \times \Omega \times \mathbb{F}_{S}^{0}(\mathbb{H}) & \mapsto \mathbb{H}, \\
D_{1}:[0, T] \times \Omega \times \mathbb{F}_{S}^{0}(\mathbb{H}) & \mapsto L_{2}(\mathbb{U}, \mathbb{H}), \\
D_{2}:[0, T] \times[0, T] \times \Omega \times \mathbb{F}_{S}^{0}(\mathbb{H}) & \mapsto L_{2}(\mathbb{U}, \mathbb{H})
\end{aligned}
$$

are progressively measurable, for example, for every $0 \leqslant t \leqslant T$, the mapping $(s, \omega, x) \mapsto$ $D_{2}(t, s, \omega, x)$ is $\mathcal{M} \times \mathcal{B}\left(\mathbb{F}_{S}^{0}(\mathbb{H})\right) / \mathcal{B}\left(L_{2}(\mathbb{U}, \mathbb{H})\right)$-measurable.

We make the following assumptions:

(HF1) $X_{0} \in L^{2}\left(\Omega, \mathcal{F}_{0}, P ; \mathbb{F}_{S}^{0}(\mathbb{H})\right)$ and $(A, 0)$ satisfies $\mathscr{H}\left(\lambda_{0}, \lambda_{1}, \lambda_{2}, \lambda_{3}, \xi, \eta_{1}, \eta_{2}, q_{1}, q_{2}\right)$, where $\lambda_{i} \in L^{1}([0, T]), i=0,1,2,3$ are non-random strictly positive functions, $q_{i} \geqslant 2$, and $0 \leqslant \eta_{i} \in L^{\frac{q_{i}}{q_{i}-1}}(\mathfrak{A}), i=1,2$, and $0 \leqslant \xi \in L^{1}(\mathfrak{A})$.

(HF2) There exist a positive real function $\lambda_{3} \in L^{1}([0, T])$ and an increasing concave function $\rho$ satisfying (3) such that for all $(s, \omega) \in[0, T] \times \Omega$ and $x, y \in \mathbb{F}_{S}^{0}$

$$
\begin{aligned}
\left\|C_{1}(s, \omega, x)-C_{1}(s, \omega, y)\right\|_{\mathbb{H}}^{2} & \leqslant \lambda_{3}(s) \cdot \rho\left(\|x-y\|_{\mathbb{F}_{S}^{0}}^{2}\right), \\
\left\|D_{1}(s, \omega, x)-D_{1}(s, \omega, y)\right\|_{L_{2}(\mathbb{U}, \mathbb{H})}^{2} & \leqslant \lambda_{3}(s) \cdot \rho\left(\|x-y\|_{\mathbb{F}_{S}^{0}}^{2}\right) .
\end{aligned}
$$

(HF3) There exists a positive real function $\lambda_{5}$ satisfying $t \mapsto \int_{0}^{t} \lambda_{5}(t, s) \mathrm{d} s \in L^{1}([0, T])$ such that for all $t, s \in[0, T], \omega \in \Omega$ and $x, y \in \mathbb{F}_{S}^{0}$

$$
\begin{aligned}
\left\|C_{2}(t, s, \omega, x)-C_{2}(t, s, \omega, y)\right\|_{\mathbb{H}}^{2} & \leqslant \lambda_{5}(t, s) \cdot \rho\left(\|x-y\|_{\mathbb{F}_{S}^{0}}^{2}\right), \\
\left\|D_{2}(t, s, \omega, x)-D_{2}(t, s, \omega, y)\right\|_{L_{2}(\mathbb{U}, \mathbb{H})}^{2} & \leqslant \lambda_{5}(t, s) \cdot \rho\left(\|x-y\|_{\mathbb{F}_{S}^{0}}^{2}\right),
\end{aligned}
$$

where $\rho$ is same as in (HF2). 
(HF4) There exist a positive progressively measurable process $\lambda_{6}$ and a positive real function $\lambda_{7}$ satisfying $\int_{0}^{t}\left[\lambda_{7}(t, s)+\mathbb{E} \lambda_{6}(t, s)\right] \mathrm{d} s \leqslant c_{0} \lambda_{2}^{2 / q_{1}}(t)$, and $0 \leqslant \zeta \in L^{1}(\mathfrak{A})$ such that for all $t, s \in[0, T], \omega \in \Omega$ and $x \in \mathbb{F}_{S}^{0}$

$$
\begin{aligned}
\left\|C_{1}(s, \omega, x)\right\|_{\mathbb{H}}^{2}+\left\|D_{1}(s, \omega, x)\right\|_{L_{2}(\mathbb{U}, \mathbb{H})}^{2} & \leqslant c_{0} \lambda_{1}^{2 / q_{1}}(s) \cdot\left(\zeta(s, \omega)+\|x\|_{\mathbb{F}_{S}^{0}}^{2}\right), \\
\left\|C_{2}(t, s, \omega, x)\right\|_{\mathbb{H}}^{2}+\left\|D_{2}(t, s, \omega, x)\right\|_{L_{2}(\mathbb{U}, \mathbb{H})} & \leqslant \lambda_{6}(t, s, \omega)+\lambda_{7}(t, s) \cdot\|x\|_{\mathbb{F}_{S}^{0}}^{2},
\end{aligned}
$$

where $\lambda_{1}$ and $q_{1}$ are same as in (HF1).

Definition 5.1. An $\mathbb{H}$-valued continuous $\mathcal{F}_{t}$-adapted process $X$ on $[-S, T]$ is called a solution of $E q \cdot(47)$, if

$$
\mathbb{E}\left(\|X\|_{\mathbb{F}_{S}^{T}}^{2}\right)+\|X .(0)\|_{\mathbb{K}_{2,1}}^{q_{1}}+\|X .(0)\|_{\mathbb{K}_{2,2}}^{q_{2}}<+\infty,
$$

and (47) holds in $\mathbb{X}^{*}$ for all $t \in[0, T]$ a.s.

Theorem 5.2. Under (HF1)-(HF4), there exists a unique solution to Eq. $\sqrt{47})$ in the sense of Definition 5.1 .

Proof. Let $X_{t}^{1} \equiv X_{0}(\cdot)$. One constructs the following iteration sequence $X_{t}^{n}$ for $n \in \mathbb{N}$ :

$$
\begin{aligned}
X_{t}^{n+1}(0)= & X_{0}(0)+\int_{0}^{t} A\left(s, X_{s}^{n+1}(0)\right) \mathrm{d} s \\
& +\int_{0}^{t} C_{1}\left(s, X_{s}^{n}\right) \mathrm{d} s+\int_{0}^{t} \int_{0}^{s} C_{2}\left(s, r, X_{r}^{n}\right) \mathrm{d} r \mathrm{~d} s \\
& +\int_{0}^{t} D_{1}\left(s, X_{s}^{n}\right) \mathrm{d} W(s)+\int_{0}^{t} \int_{0}^{s} D_{2}\left(s, r, X_{r}^{n}\right) \mathrm{d} W_{r} \mathrm{~d} s \\
=: & X_{0}(0)+\int_{0}^{t} A^{n}\left(s, X_{s}^{n+1}(0)\right) \mathrm{d} s+\int_{0}^{t} B^{n}(s) \mathrm{d} W(s),
\end{aligned}
$$

where

$$
\begin{aligned}
A^{n}(s, x) & :=A_{1}^{n}(s, x)+A_{2}(s, x) \in \mathbb{X}^{*} \\
A_{1}^{n}(s, x) & :=A_{1}(s, x)+G^{n}(s) \mathbb{X}_{1}^{*} \\
B^{n}(s) & :=D_{1}\left(s, X_{s}^{n}\right) \in L_{2}(\mathbb{U}, \mathbb{H})
\end{aligned}
$$

and

$$
G^{n}(s):=C_{1}\left(s, X_{s}^{n}\right)+\int_{0}^{s} C_{2}\left(s, r, X_{r}^{n}\right) \mathrm{d} r+\int_{0}^{s} D_{2}\left(s, r, X_{r}^{n}\right) \mathrm{d} W_{r} .
$$

First of all, we clearly have for any $x, y \in \mathbb{X}$ and $s \in[0, T]$

$$
2\left[x-y, A^{n}(s, x)-A^{n}(s, y)\right]_{\mathbb{X}} \leqslant \lambda_{0}(s) \cdot\|x-y\|_{\mathbb{H}}^{2} .
$$

Secondly, by (H3) we have for any $x \in \mathbb{X}$ and $s \in[0, T]$

$$
\begin{aligned}
& 2\left[x, A^{n}(s, x)\right]_{\mathbb{X}}+\left\|B^{n}(s)\right\|_{L_{2}(\mathbb{U}, \mathbb{H})}^{2} \\
= & 2[x, A(s, x)]_{\mathbb{X}}+2\left\langle x, G^{n}(s)\right\rangle_{\mathbb{H}}+\left\|D_{1}\left(s, X_{s}^{n}\right)\right\|_{L_{2}(\mathbb{U}, \mathbb{H})}^{2} \\
\leqslant & -\sum_{i=1,2}\left(\lambda_{i}(s) \cdot\|x\|_{\mathbb{X}_{i}}^{q_{i}}\right)+\left(\lambda_{3}(s)+1\right) \cdot\|x\|_{\mathbb{H}}^{2}+\xi(s) \\
& +\left\|G^{n}(s)\right\|_{\mathbb{H}}^{2}+\left\|D_{1}\left(s, X_{s}^{n}\right)\right\|_{L_{2}(\mathbb{U}, \mathbb{H})}^{2} .
\end{aligned}
$$

Moreover, by the embedding $\mathbb{H} \subset \mathbb{X}_{1}^{*}$ and (H4) we have for any $x \in \mathbb{X}$ and $s \in[0, T]$

$$
\left\|A_{1}^{n}(s, x)\right\|_{\mathbb{X}_{1}^{*}} \leqslant\left\|A_{1}(s, x)\right\|_{\mathbb{X}_{1}^{*}}+\underset{28}{\| G^{n}(s)} \|_{\mathbb{X}_{1}^{*}}
$$




$$
\leqslant \eta_{1}(s) \cdot \lambda_{1}^{1 / q_{1}}(s)+c_{A_{1}} \cdot \lambda_{1}(s, \omega) \cdot\|x\|_{\mathbb{X}_{1}}^{q_{1}-1}+c_{0}\left\|G^{n}(s)\right\|_{\mathbb{H}} \cdot
$$

Hence, $\left(A^{n}, B^{n}\right)$ satisfies $\mathscr{H}\left(\lambda_{0}, \lambda_{1}, \lambda_{2}, \lambda_{3}+1, \xi^{n}, \eta_{1}^{n}, \eta_{2}, q_{1}, q_{2}\right)$, where

$$
\begin{aligned}
\xi^{n}(s) & :=\xi(s)+\left\|D_{1}\left(s, X_{s}^{n}\right)\right\|_{L_{2}(\mathbb{U}, \mathbb{H})}^{2}+\left\|G^{n}(s)\right\|_{\mathbb{H}}^{2} \\
\eta_{1}^{n}(s) & :=\eta_{1}(s)+c_{0} \cdot \lambda_{1}^{-1 / q_{1}}(s) \cdot\left\|G^{n}(s)\right\|_{\mathbb{H}} .
\end{aligned}
$$

Noting that by (HF4)

$$
\begin{aligned}
\mathbb{E}\left\|C_{1}\left(s, X_{s}^{n}\right)\right\|_{\mathbb{H}}^{2}+\mathbb{E}\left\|D_{1}\left(s, X_{s}^{n}\right)\right\|_{L_{2}(\mathbb{U}, \mathbb{H})}^{2} \leqslant c_{0} \lambda_{2}^{2 / q_{1}}(s)\left(\mathbb{E} \zeta(s)+\mathbb{E}\left\|X_{s}^{n}\right\|_{\mathbb{F}_{S}^{0}}^{2}\right) \\
\mathbb{E}\left\|\int_{0}^{s} C_{2}\left(s, r, X_{r}^{n}\right) \mathrm{d} r\right\|_{\mathbb{H}}^{2} \leqslant c_{0} \lambda_{2}^{2 / q_{1}}(s)\left(1+\mathbb{E}\left\|X^{n}\right\|_{\mathbb{F}_{S}^{s}}^{2}\right) \\
\mathbb{E}\left\|\int_{0}^{s} D_{2}\left(s, r, X_{r}^{n}\right) \mathrm{d} W_{r}\right\|_{\mathbb{H}}^{2} \leqslant c_{0} \lambda_{2}^{2 / q_{1}}(s)\left(1+\mathbb{E}\left\|X^{n}\right\|_{\mathbb{F}_{S}^{s}}^{2}\right),
\end{aligned}
$$

we have by $q_{1} \geqslant 2$ and Young's inequality (5)

$$
\begin{aligned}
\mathbb{E}\left(\left\|G^{n}(s)\right\|_{\mathbb{H}}^{\frac{q_{1}}{q_{1}-1}}\right) & \leqslant\left(\mathbb{E}\left\|G^{n}(s)\right\|_{\mathbb{H}}^{2}\right)^{\frac{q_{1}}{2\left(q_{1}-1\right)}} \\
& \leqslant c_{0} \cdot \lambda_{1}^{\frac{1}{q_{1}-1}}(s) \cdot\left(1+\mathbb{E}\left\|X^{n}\right\|_{\mathbb{F}_{S}^{s}}^{2}\right)^{\frac{q_{1}}{2\left(q_{1}-1\right)}} \\
& \leqslant c_{0} \cdot \lambda_{1}^{\frac{1}{q_{1}-1}}(s) \cdot\left(1+\mathbb{E}\left\|X^{n}\right\|_{\mathbb{F}_{S}^{s}}^{2}\right) .
\end{aligned}
$$

Therefore,

$$
\begin{aligned}
\int_{0}^{t} \mathbb{E} \xi^{n}(s) \mathrm{d} s & \leqslant c_{0}+c_{0} \int_{0}^{t}\left(\lambda_{1}(s)+1\right) \cdot \mathbb{E}\left\|X_{s}^{n}\right\|_{\mathbb{F}_{S}^{0}}^{2} \mathrm{~d} s \\
\int_{0}^{t} \mathbb{E}\left(\left|\eta_{1}^{n}(s)\right|^{\frac{q_{1}}{q_{1}-1}}\right) \mathrm{d} s & \leqslant c_{0}+c_{0} \int_{0}^{t} \mathbb{E}\left\|X^{n}\right\|_{\mathbb{F}_{S}^{s}}^{2} \mathrm{~d} s .
\end{aligned}
$$

Thus, by Theorem 3.5 we have

$$
\begin{aligned}
\mathbb{E}\left(\left\|X_{\cdot}^{n+1}(0)\right\|_{\mathbb{F}_{0}^{t}}^{2}\right) & \leqslant c_{0}\left(\mathbb{E}\left\|X_{0}(0)\right\|_{\mathbb{H}}^{2}+\int_{0}^{t} \mathbb{E}\left(\xi^{n}(s)+\left|\eta_{1}^{n}(s)\right|^{\frac{q_{1}}{q_{1}-1}}+\left|\eta_{2}(s)\right|^{\frac{q_{2}}{q_{2}-1}}\right) \mathrm{d} s\right) \\
& \leqslant c_{0}+c_{0} \int_{0}^{t}\left(\lambda_{1}(s)+1\right) \mathbb{E}\left\|X^{n}\right\|_{\mathbb{F}_{S}^{s}}^{2} \mathrm{~d} s
\end{aligned}
$$

where $c_{0}$ is independent of $n$.

By induction methods and Theorem 3.6. $\left\{X_{t}^{n}, n \in \mathbb{N}\right\}$ are thus well defined. Moreover, by Theorem 3.5 we have

$$
\left\|X^{n}(0)\right\|_{\mathbb{K}_{2,1}}^{q_{1}}+\left\|X^{n}(0)\right\|_{\mathbb{K}_{2,2}}^{q_{2}} \leqslant c_{n}<+\infty
$$

Setting

$$
g_{n}(t):=\sup _{k \leqslant n+1} \mathbb{E}\left(\left\|X^{k}\right\|_{\mathbb{F}_{S}^{t}}^{2}\right)
$$

we then have by (48)

$$
\begin{aligned}
g_{n}(t) & \leqslant 2 \sup _{k \leqslant n+1} \mathbb{E}\left(\left\|X_{.}^{k}(0)\right\|_{\mathbb{F}_{0}^{t}}^{2}\right)+2 \mathbb{E}\left\|X_{0}\right\|_{\mathbb{F}_{S}^{0}}^{2} \\
& \leqslant c_{0}+c_{0} \int_{0}^{t}\left(\lambda_{1}(s)+1\right) g_{n}(s) d s
\end{aligned}
$$


Applying Gronwall's inequality yields

$$
\sup _{n \in \mathbb{N}} \mathbb{E}\left(\sup _{t \in[0, T]}\left\|X_{t}^{n}\right\|_{\mathbb{F}_{S}^{0}}^{2}\right)=\sup _{n \in \mathbb{N}} \mathbb{E}\left(\left\|X^{n}\right\|_{\mathbb{F}_{S}^{T}}^{2}\right)<+\infty .
$$

Next, set $Z_{t}^{n, m}:=X_{t}^{n}-X_{t}^{m}$. By Itô's formula, (H2) and (HF2), we have

$$
\begin{aligned}
\left\|Z_{t}^{n+1, m+1}(0)\right\|_{\mathbb{H}}^{2}= & 2 \int_{0}^{t}\left[Z_{s}^{n+1, m+1}(0), A^{n}\left(s, X_{s}^{n+1}(0)\right)-A^{m}\left(s, X_{s}^{m+1}(0)\right)\right]_{\mathbb{X}} \mathrm{d} s \\
& +2 \int_{0}^{t}\left\langle Z_{s}^{n+1, m+1}(0),\left(B^{n}(s)-B^{m}(s)\right) \mathrm{d} W(s)\right\rangle_{\mathbb{H}} \\
& +\int_{0}^{t}\left\|B^{n}(s)-B^{m}(s)\right\|_{L_{2}(\mathbb{U}, \mathbb{H})}^{2} \mathrm{~d} s \\
= & 2 \int_{0}^{t}\left[Z_{s}^{n+1, m+1}(0), A\left(s, X_{s}^{n+1}(0)\right)-A\left(s, X_{s}^{m+1}(0)\right)\right]_{\mathbb{X}} \mathrm{d} s \\
& +\int_{0}^{t}\left\|D_{1}\left(s, X_{s}^{n}\right)-D_{1}\left(s, X_{s}^{m}\right)\right\|_{L_{2}(\mathbb{U}, \mathbb{H})}^{2} \mathrm{~d} s+\sum_{i=1}^{4} I_{t}^{i} \\
\leqslant & \int_{0}^{t} \lambda_{0}(s) \cdot\left\|Z_{s}^{n+1, m+1}(0)\right\|_{\mathbb{H}}^{2} \mathrm{~d} s \\
& +\int_{0}^{t} \lambda_{3}(s) \cdot \rho\left(\left\|Z_{s}^{n, m}\right\|_{\mathbb{F}_{S}^{0}}^{2}\right) \mathrm{d} s+\sum_{i=1}^{4} I_{t}^{i},
\end{aligned}
$$

where

$$
\begin{aligned}
I_{t}^{1} & :=2 \int_{0}^{t}\left\langle Z_{s}^{n+1, m+1}(0), C_{1}\left(s, X_{s}^{n}\right)-C_{1}\left(s, X_{s}^{m}\right)\right\rangle_{\mathbb{H}} \mathrm{d} s \\
I_{t}^{2} & :=2 \int_{0}^{t}\left\langle Z_{s}^{n+1, m+1}(0), \int_{0}^{s} C_{2}\left(s, r, X_{r}^{n}\right)-C_{2}\left(s, r, X_{r}^{m}\right) \mathrm{d} r\right\rangle_{\mathbb{H}} \mathrm{d} s \\
I_{t}^{3} & :=2 \int_{0}^{t}\left\langle Z_{s}^{n+1, m+1}(0),\left(D_{1}\left(s, X_{s}^{n}\right)-D_{1}\left(s, X_{s}^{m}\right)\right) \mathrm{d} W(s)\right\rangle_{\mathbb{H}} \\
I_{t}^{4} & :=2 \int_{0}^{t}\left\langle Z_{s}^{n+1, m+1}(0), \int_{0}^{s}\left(D_{2}\left(s, r, X_{r}^{n}\right)-D_{2}\left(s, r, X_{r}^{m}\right)\right) \mathrm{d} W_{r}\right\rangle_{\mathbb{H}} \mathrm{d} s .
\end{aligned}
$$

By Burkholder's inequality and Young's inequality (5), we have

$$
\begin{aligned}
\mathbb{E}\left(\left\|I_{.}^{3}\right\|_{\mathbb{F}_{0}^{t}}\right) & \leqslant c_{0} \mathbb{E}\left(\int_{0}^{t}\left\|Z_{s}^{n+1, m+1}(0)\right\|_{\mathbb{H}}^{2} \cdot\left\|D_{1}\left(s, X_{s}^{n}\right)-D_{1}\left(s, X_{s}^{m}\right)\right\|_{L_{2}(\mathbb{U}, \mathbb{H})}^{2} \mathrm{~d} s\right)^{1 / 2} \\
& \leqslant \frac{1}{2} \mathbb{E}\left\|Z^{n+1, m+1}(0)\right\|_{\mathbb{F}_{0}^{t}}^{2}+c_{0} \int_{0}^{t} \lambda_{3}(s) \cdot \mathbb{E} \rho\left(\left\|Z_{s}^{n, m}\right\|_{\mathbb{F}_{S}^{0}}^{2}\right) \mathrm{d} s
\end{aligned}
$$

and

$$
\begin{aligned}
\mathbb{E}\left(\left\|I_{.}^{4}\right\|_{\mathbb{F}_{0}^{t}}\right) & \leqslant \int_{0}^{t} \mathbb{E}\left\|Z_{s}^{n+1, m+1}(0)\right\|_{\mathbb{H}^{2}}^{2} \mathrm{~d} s+\int_{0}^{t} \int_{0}^{s} \lambda_{5}(s, r) \cdot \mathbb{E} \rho\left(\left\|Z_{r}^{n, m}\right\|_{\mathbb{F}_{S}^{0}}^{2}\right) \mathrm{d} r \mathrm{~d} s \\
& \leqslant \int_{0}^{t} \mathbb{E}\left\|Z^{n+1, m+1}(0)\right\|_{\mathbb{F}_{0}^{s}}^{2} \mathrm{~d} s+\int_{0}^{t} \mathbb{E} \rho\left(\left\|Z^{n, m}\right\|_{\mathbb{F}_{0}^{s}}^{2}\right)\left(\int_{0}^{s} \lambda_{5}(s, r) \mathrm{d} r\right) \mathrm{d} s,
\end{aligned}
$$


where we have used that $\rho$ is increasing and

$$
\sup _{r \in[0, s]}\left\|Z_{r}^{n, m}\right\|_{\mathbb{F}_{S}^{0}}^{2}=\left\|Z^{n, m}\right\|_{\mathbb{F}_{0}^{s}}^{2} .
$$

Similarly,

$$
\begin{aligned}
& \mathbb{E}\left(\left\|I_{.}^{1}\right\|_{\mathbb{F}_{0}^{t}}\right) \leqslant \int_{0}^{t} \mathbb{E}\left\|Z^{n+1, m+1}(0)\right\|_{\mathbb{F}_{0}^{s}}^{2} \mathrm{~d} s+\int_{0}^{t} \lambda_{3}(s) \cdot \mathbb{E} \rho\left(\left\|Z^{n, m}\right\|_{\mathbb{F}_{0}^{s}}^{2}\right) \mathrm{d} s, \\
& \mathbb{E}\left(\left\|I_{.}^{2}\right\|_{\mathbb{F}_{0}^{t}}\right) \leqslant \int_{0}^{t} \mathbb{E}\left\|Z^{n+1, m+1}(0)\right\|_{\mathbb{F}_{0}^{s}}^{2} \mathrm{~d} s+\int_{0}^{t} \mathbb{E} \rho\left(\left\|Z^{n, m}\right\|_{\mathbb{F}_{0}^{s}}^{2}\right)\left(\int_{0}^{s} \lambda_{5}(s, r) \mathrm{d} r\right) \mathrm{d} s .
\end{aligned}
$$

Combining the above calculations, we obtain

$$
\begin{aligned}
\mathbb{E}\left\|Z^{n+1, m+1}(0)\right\|_{\mathbb{F}_{0}^{t}}^{2} \leqslant & 2 \int_{0}^{t}\left(\lambda_{0}(s)+3\right) \cdot \mathbb{E}\left\|Z^{n+1, m+1}(0)\right\|_{\mathbb{F}_{0}^{s}}^{2} \mathrm{~d} s \\
& +c_{0} \int_{0}^{t} \lambda_{8}(s) \cdot \mathbb{E}\left(\rho\left(\left\|Z^{n, m}\right\|_{\mathbb{F}_{0}^{s}}^{2}\right)\right) \mathrm{d} s,
\end{aligned}
$$

where $\lambda_{8}(s):=\lambda_{3}(s)+\int_{0}^{s} \lambda_{5}(s, r) \mathrm{d} r$.

By Gronwall's inequality and Jensen's inequality, we have

$$
\mathbb{E}\left\|Z^{n+1, m+1}(0)\right\|_{\mathbb{F}_{0}^{t}}^{2} \leqslant c_{0} \int_{0}^{t} \lambda_{8}(s) \cdot \rho\left(\mathbb{E}\left(\left\|Z^{n, m}\right\|_{\mathbb{F}_{0}^{s}}^{2}\right)\right) \mathrm{d} s .
$$

Now setting

$$
g(t):=\limsup _{n, m \rightarrow \infty} \mathbb{E}\left\|Z^{n+1, m+1}(0)\right\|_{\mathbb{F}_{0}^{t}}^{2}=\limsup _{n, m \rightarrow \infty} \mathbb{E}\left\|Z^{n+1, m+1}\right\|_{\mathbb{F}_{0}^{t}}^{2},
$$

we then get by (49) and Fatou's lemma

$$
g(t) \leqslant c_{0} \int_{0}^{t} \lambda_{8}(s) \cdot \rho(g(s)) \mathrm{d} s .
$$

Using Lemma 2.2 yields that

$$
g(t)=0 \text {. }
$$

Therefore, there is an $\mathbb{H}$-valued continuous adapted process $X$ such that

$$
\lim _{n \rightarrow \infty} \mathbb{E}\left\|X^{n}-X\right\|_{\mathbb{F}_{0}^{T}}^{2}=0
$$

It remains to show that $X_{t}$ is a solution in the sense of Definition 5.1, Let $\tilde{X}(t)$ solve the following equation(Theorem 3.6)

$$
\begin{aligned}
\tilde{X}(t)= & X_{0}+\int_{0}^{t} A(s, \tilde{X}(s)) \mathrm{d} s+\int_{0}^{t} C_{1}\left(s, X_{s}\right) \mathrm{d} s+\int_{0}^{t} \int_{0}^{s} C_{2}\left(s, r, X_{r}\right) \mathrm{d} r \mathrm{~d} s \\
& +\int_{0}^{t} D_{1}\left(s, s, X_{s}\right) \mathrm{d} W(s)+\int_{0}^{t} \int_{0}^{s} D_{2}\left(s, r, X_{r}\right) \mathrm{d} W_{r} \mathrm{~d} s .
\end{aligned}
$$

As in estimating (50), we can prove that

$$
\mathbb{E}\left\|X_{.}^{n+1}(0)-\tilde{X}(\cdot)\right\|_{\mathbb{F}_{0}^{t}}^{2} \leqslant c_{0} \int_{0}^{t} \lambda_{8}(s) \cdot \rho\left(\mathbb{E}\left\|X^{n}-X .(0)\right\|_{\mathbb{F}_{0}^{s}}^{2}\right) \mathrm{d} s .
$$

Taking limits and by the dominated convergence theorem, we get

$$
\lim _{n \rightarrow \infty} \mathbb{E}\left\|X^{n+1}(0)-\tilde{X}(\cdot)\right\|_{\mathbb{F}_{0}^{t}}^{2}=0
$$

So, for each $t \in[0, T]$

$$
X(t)=\underset{31}{\tilde{X}(t), \quad \text { a.s.. }}
$$


Moreover, by Theorems 3.6 and 3.5, for almost all $\omega, t \mapsto \tilde{X}(t, \omega)$ is continuous in $\mathbb{H}$, and

$$
\|\tilde{X} .(0)\|_{\mathbb{K}_{2,1}}^{q_{1}}+\|\tilde{X} .(0)\|_{\mathbb{K}_{2,2}}^{q_{2}}<+\infty \text {. }
$$

The uniqueness follows from the similar calculations, and the proof is thus complete.

We now consider the following stochastic Volterra evolution equation:

$$
X_{t}(0)=X_{0}(0)+\int_{0}^{t} A\left(s, X_{s}\right) \mathrm{d} s+\int_{0}^{t} C\left(t, s, X_{s}\right) \mathrm{d} s+\int_{0}^{t} D\left(t, s, X_{s}\right) \mathrm{d} W_{s},
$$

where $X_{0}$ is an $\mathcal{F}_{0}$-measurable $\mathbb{F}_{S}^{0}(\mathbb{H})$-valued random variable and $A=A_{1}+A_{2}$,

$$
\begin{aligned}
A_{i}:[0, T] \times \Omega \times \mathbb{X}_{i} & \mapsto \mathbb{X}_{i}^{*}, \quad i=1,2, \\
C:[0, T] \times[0, T] \times \Omega \times \mathbb{F}_{S}^{0}(\mathbb{H}) & \mapsto \mathbb{H}, \\
D:[0, T] \times[0, T] \times \Omega \times \mathbb{F}_{S}^{0}(\mathbb{H}) & \mapsto L_{2}(\mathbb{U}, \mathbb{H})
\end{aligned}
$$

are progressively measurable.

We make the following assumptions:

(HV1) $X_{0} \in L^{2}\left(\Omega, \mathcal{F}_{0}, P ; \mathbb{F}_{S}^{0}(\mathbb{H})\right)$ and $(A, 0)$ satisfies $\mathscr{H}\left(\lambda_{0}, \lambda_{1}, \lambda_{2}, \lambda_{3}, \xi, \eta_{1}, \eta_{2}, q_{1}, q_{2}\right)$, where $\lambda_{i} \in L^{1}([0, T]), i=0,1,2,3$ are non-random strictly positive functions, $q_{i} \geqslant 2$, and $0 \leqslant \eta_{i} \in L^{\frac{q_{i}}{q_{i}-1}}(\mathfrak{A}), i=1,2$, and $0 \leqslant \xi \in L^{1}(\mathfrak{A})$.

(HV2) $C(\cdot, s, \omega, x)$ and $D(\cdot, s, \omega, x)$ are differentiable with respect to the first variable for all $s, \omega, x$, and there exist a positive real function $\lambda_{5}$ satisfying $t \mapsto \int_{0}^{t} \lambda_{5}(t, s) \mathrm{d} s \in$ $L^{1}([0, T])$ and an increasing concave function $\rho$ satisfying (3) such that for all $t, s \in[0, T], \omega \in \Omega$ and $x, y \in \mathbb{F}_{S}^{0}$

$$
\begin{aligned}
\left\|\partial_{t} C(t, s, \omega, x)-\partial_{t} C(t, s, \omega, y)\right\|_{\mathbb{H}}^{2} & \leqslant \lambda_{5}(t, s) \cdot \rho\left(\|x-y\|_{\mathbb{F}_{S}^{0}}^{2}\right), \\
\left\|\partial_{t} D(t, s, \omega, x)-\partial_{t} D(t, s, \omega, y)\right\|_{L_{2}(\mathbb{U}, \mathbb{H})}^{2} & \leqslant \lambda_{5}(t, s) \cdot \rho\left(\|x-y\|_{\mathbb{F}_{S}^{0}}^{2}\right) .
\end{aligned}
$$

(HV3) There exist a positive real function $\lambda_{3} \in L^{1}([0, T])$ such that for all $(s, \omega) \in$ $[0, T] \times \Omega$ and $x, y \in \mathbb{F}_{S}^{0}$

$$
\begin{aligned}
\|C(s, s, \omega, x)-C(s, s, \omega, y)\|_{\mathbb{H}}^{2} & \leqslant \lambda_{3}(s) \cdot \rho\left(\|x-y\|_{\mathbb{F}_{S}^{0}}^{2}\right), \\
\|D(s, s, \omega, x)-D(s, s, \omega, y)\|_{L_{2}(\mathbb{U}, \mathbb{H})}^{2} & \leqslant \lambda_{3}(s) \cdot \rho\left(\|x-y\|_{\mathbb{F}_{S}^{0}}^{2}\right),
\end{aligned}
$$

where $\rho$ is same as in (HV2).

(HV4) There exist a positive progressively measurable process $\lambda_{6}$ and a positive real function $\lambda_{7}$ satisfying $\int_{0}^{t}\left[\lambda_{7}(t, s)+\mathbb{E} \lambda_{6}(t, s)\right] \mathrm{d} s \leqslant c_{0} \lambda_{2}^{2 / q_{1}}(t)$, and $0 \leqslant \zeta \in L^{1}(\mathfrak{A})$ such that for all $t, s \in[0, T], \omega \in \Omega$ and $x \in \mathbb{F}_{S}^{0}$

$$
\begin{aligned}
\|C(s, s, \omega, x)\|_{\mathbb{H}}^{2}+\|D(s, s, \omega, x)\|_{L_{2}(\mathbb{U}, \mathbb{H})}^{2} & \leqslant c_{0} \lambda_{2}^{2 / q_{1}}(s) \cdot\left(\zeta(s, \omega)+\|x\|_{\mathbb{F}_{S}^{0}}^{2}\right), \\
\left\|\partial_{t} C(t, s, \omega, x)\right\|_{\mathbb{H}}^{2}+\left\|\partial_{t} D(t, s, \omega, x)\right\|_{L_{2}(\mathbb{U}, \mathbb{H})}^{2} & \leqslant \lambda_{6}(t, s, \omega)+\lambda_{7}(t, s) \cdot\|x\|_{\mathbb{F}_{S}^{0}}^{2},
\end{aligned}
$$

where $\lambda_{1}$ and $q_{1}$ are same as in $(\mathbf{H V} \mathbf{1})$.

Definition 5.3. An $\mathbb{H}$-valued continuous $\mathcal{F}_{t}$-adapted process $X$ on $[-S, T]$ is called a solution of Eq.(51), if

$$
\mathbb{E}\left(\|X\|_{\mathbb{F}_{S}^{T}}^{2}\right)+\|X .(0)\|_{\mathbb{K}_{2,1}}^{q_{1}}+\|X .(0)\|_{\mathbb{K}_{2,2}}^{q_{2}}<+\infty,
$$

and (51) holds in $\mathbb{X}^{*}$ for all $t \in[0, T]$ a.s..

Theorem 5.4. Under (HV1)-(HV4), there exists a unique solution to Eq. (51) in the sense of Definition 5.3. 
Proof. Noting that by stochastic Fubini's theorem, we have

$$
\begin{aligned}
\int_{0}^{t} D\left(t, s, X_{s}\right) \mathrm{d} W_{s} & =\int_{0}^{t}\left(D\left(t, s, X_{s}\right)-D\left(s, s, X_{s}\right)\right) \mathrm{d} W_{s}+\int_{0}^{t} D\left(s, s, X_{s}\right) \mathrm{d} W_{s} \\
& =\int_{0}^{t} \int_{s}^{t} \partial_{r} D\left(r, s, X_{s}\right) \mathrm{d} r \mathrm{~d} W_{s}+\int_{0}^{t} D\left(s, s, X_{s}\right) \mathrm{d} W_{s} \\
& =\int_{0}^{t} \int_{0}^{s} \partial_{s} D\left(s, r, X_{r}\right) \mathrm{d} W_{r} \mathrm{~d} s+\int_{0}^{t} D\left(s, s, X_{s}\right) \mathrm{d} W_{s}
\end{aligned}
$$

and

$$
\int_{0}^{t} C\left(t, s, X_{s}\right) \mathrm{d} s=\int_{0}^{t} \int_{0}^{s} \partial_{s} C\left(s, r, X_{r}\right) \mathrm{d} r \mathrm{~d} s+\int_{0}^{t} C\left(s, s, X_{s}\right) \mathrm{d} s .
$$

Solving Eq. (51) is then equivalent to solving the following equation

$$
\begin{aligned}
X_{t}= & X_{0}+\int_{0}^{t} A\left(s, X_{s}(0)\right) \mathrm{d} s+\int_{0}^{t} C\left(s, s, X_{s}\right) \mathrm{d} s+\int_{0}^{t} \int_{0}^{s} \partial_{s} C\left(s, r, X_{r}\right) \mathrm{d} r \mathrm{~d} s \\
& +\int_{0}^{t} D\left(s, s, X_{s}\right) \mathrm{d} W_{s}+\int_{0}^{t} \int_{0}^{s} \partial_{s} D\left(s, r, X_{r}\right) \mathrm{d} W_{r} \mathrm{~d} s .
\end{aligned}
$$

The result now follows from Theorem 5.2 .

\section{Acknowledgements:}

This work was done while the author was a fellow of Alexander-Humboldt Foundation. He would like to thank Professor Michael Röckner, his host in Bielefeld University, for providing him his Lecture Notes [26] and a stimulating environment. He is also grateful to Professor Jiagang Ren, Dr. Wei Liu and Dr. Huijie Qiao for their valuable discussions and suggestions.

\section{REFERENCES}

[1] Briand, Ph. and Carmona R.: BSDEs with polynomial growth generators. J. Appl. Math. Stochastic Anal. 13 (2000), 207-238.

[2] Barbu, V.: Nonlinear semigroups and differential equations in Banach spaces. Noordhoff International Publishing, Leyden The Netherlands, 1976.

[3] Berger, M. and Mizel, V.: Volterra equations with Itô integrals, I and Ш, J. Int. Equation, 2 (1980), pp. 187-245, 319-337.

[4] Bihari, I.: A generalization of a lemma of Belmman and its application to uniqueness problem of differential equations, Acta. Math., Acad. Sci. Hungar, 7 (1956), pp. 71-94.

[5] Brezis, H.: Opérateurs monotones et semi-groupes de contractions das les espaces de Hilbert. NorthHolland, Amsterdam, 1973.

[6] Brzeźniak, Z.: Stochastic partial differential equations in M-type 2 Banach spaces. Potential Anal. 4 (1995), no. 1, 1-45.

[7] Brzeźniak, Z.: On stochastic convolution in Banach spaces and applications. Stochastics Stochastics Rep. 61 (1997), no. 3-4, 245-295.

[8] Da Prato, G. and Röckner, M.: Weak solutions to stochastic porous media equations, J. Evolution Equ. 4(2004), 249-271.

[9] Da Prato, G. and Zabczyk J.: Stochastic equations in infinite dimensions. Cambridge: Cambridge University Press, 1992.

[10] Gyöngy, I.: On stochastic equations with respect to semimartingales III. Stochastic, 7(1982), 231254.

[11] Gyöngy, I. and Millet, A.: On Discretization Schemes for Stochastic Evolution Equations. Potential Analysis, (2005)23:99-134.

[12] Gyöngy, I. and Krylov, N.V.: On stochastic equations with respect to semimartingales II. Ito formula in Banach spaces. Stochastic, 6(1982), 153-173. 
[13] Hu, Y., Ma, J., Yong, J.: On semi-linear degenerate backward stochastic partial differential equations. Probab. Theory Related Fields 123 (2002), no. 3, 381-411.

[14] Krylov, N. V.: A simple proof of the existence of a solution to the Itô equation with monotone coefficients. Theory Probab. Appl. 35 (1990), no. 3, 583-587

[15] Krylov, N. V.: An analytic approach to SPDEs, in Stochastic Partial Differential Equations: Six Perspectives, Mathematical Surveys and Monographs, Vol. 64, pp. 185-242, AMS, Providence, 1999.

[16] Krylov, N.V. and Rozovskii, B.L. Stochastic evolution equations. J. Soviet Math.(Russian), 1979, pp. 71-147, Transl. 16(1981), 1233-1277.

[17] Mao, X.: Adapted solutions of backward stochastic differential equatios with non-Lipschitz coefficients. Stoch. Proc. Appl., 58(1995)281-292.

[18] Mohammend, S.E.: Stochastic Functional Differential Equations. Research Notes in Mathematics, No.99, Pitman, Boston, 1984.

[19] Pardoux, E: Stochastic partial differential equations and filtering of diffusion processes. Stochastic. 1979, 127-167.

[20] Pardoux, E.: Equations aux dérivées partielles stochastiques non lineaires monotones: Etude de solutions fortes de type Ito, Thése Doct. Sci. Math. Univ. Paris Sud. 1975.

[21] Pardoux, E. and Peng S.: Adapted solutions of backward stochastic equations, System and Control Letters, 14, 55-61, 1990.

[22] Protter, P.: Volterra equations driven by semimartingales. Ann. Probability, 13 (1985), pp. 519-530.

[23] Oksendal, B., Proske, F. and Zhang T.: Backward Stochastic Partial differential Equations with Jumps and Application to Optimal Control of Random Jump Fields, preprint.

[24] Ren, J., Röckner, M. and Wang, F.: Stochastic Generalized Porous Media and Fast Diffusion Equations. Preprint.

[25] Revuz, D. and Yor, M.: Continuous martingales and Brownian motion, Grund. Math. Wiss., 293, Springer-Verlag, 1991.

[26] Röckner, M.: Introduction to Stochastic Partial Differential Equations. Lecture Notes in Purdue University, 2006.

[27] Rozovskii, B. L.: Stochastic evolution systems. Linear theory and applications to nonlinear filtering. Mathematics and its Applications (Soviet Series), 35, Kluwer Academic Publishers, 1990.

[28] Showalter, R.E.: Monotone Operators in Banach Space and Nonlinear Partial Differential Equations, AMS, Math. Surveys and Monographs, Vol.49, 1997.

[29] Taniguchi, T., Liu, K. and Truman, A.: Existence, Uniqueness, and Asymptotic Behavior of Mild Solutions to Stochastic Functional Differential Equations in Hilbert Spaces. Journal of Differential Equations, 181, 72-91(2002).

[30] Walsh, J.B.: An introduction to stochastic partial differential equations, Lecture Notes in Math., 1180 springer, 1986, pp. 266-437.

[31] Wang, Z. and Zhang, X.: Existence-Uniqueness of Solutions to Stochastic Volterra Equations with Singular Kernels and Non-Lipschitz Coefficients. Preprint.

[32] Yamada, T., Watanabe, S.: On the uniquenss of solutions of stochastic differential equations, J. Math. Kyoto, Univ., 11 (1971), 553-563.

[33] Zeidler, E.: Nonlinear functional analysis and its applications, Vol. II(A, B), Springer-Verlag, New York, 1990.

[34] Zhang, X.: $L^{p}$-Theory of Semi-linear SPDEs on General Measure Spaces and Applications. J. Func. Anal., Vol. 239/1 pp 44-75(2006).

[35] Zhang, X.: Skorohod problem and multivalued stochastic evolution equations in Banach spaces. Bull. Sci. Math. France, (2006) in press.

[36] Zhang, X. and Zhu, J.: Non-Lipschitz stochastic differential equations driven by multi-parameter Brownian motions. Stochastic and Dynamic, Vol. 6, No. 3, 329-340(2006). 